\title{
Recursive credibility formula for chain ladder factors and the claims development result
}

\section{Journal Article}

\section{Author(s):}

Bühlmann, Hans; De Felice, Massimo; Gisler, Alois; Moriconi, Franco; Wüthrich, Mario V.

Publication date:

2009-05

Permanent link:

https://doi.org/10.3929/ethz-b-000020798

Rights / license:

In Copyright - Non-Commercial Use Permitted

Originally published in:

ASTIN Bulletin 39(1), https://doi.org/10.2143/AST.39.1.2038065 


\title{
RECURSIVE CREDIBILITY FORMULA FOR CHAIN LADDER FACTORS AND THE CLAIMS DEVELOPMENT RESULT
}

\author{
BY \\ Hans Bühlmann, Massimo De Felice, Alois Gisler, \\ FRANCO MORICONI AND MARIO V. WÜTHRICH
}

\begin{abstract}
In recent Solvency II considerations much effort has been put into the development of appropriate models for the study of the one-year loss reserving uncertainty in non-life insurance. In this article we derive formulas for the conditional mean square error of prediction of the one-year claims development result in the context of the Bayes chain ladder model studied in Gisler-Wüthrich [9]. The key to these formulas is a recursive representation for the results obtained in Gisler-Wüthrich [9].
\end{abstract}

\section{KEYWORDS}

Claims reserving, chain ladder method, credibility chain ladder method, claims development result, year end expectation, loss experience prior accident years, liability at maturity, solvency, mean square error of prediction.

\section{INTRODUCTION}

In the classical chain ladder model the parameters are assumed to be deterministic. In general, these model parameters are not known and need to be estimated from the data, see Mack [12] for the distribution-free chain ladder approach and its chain ladder factor estimators. In Gisler-Wüthrich [9] we have presented a Bayesian approach assuming that the unknown model parameters follow a prior distribution. This prior distribution indicates our uncertainty about the true parameters and allows for determining these parameters using Bayesian inference methods. One of the advantages of this Bayesian approach is that it leads to a natural and unified way for the consideration of the prediction uncertainty, that is, also the parameter estimation uncertainty is contained within the model in a natural way (see also the discussion in Section 3.2.3 in WüthrichMerz [6]). In the present manuscript we revisit the Bayesian approach presented in Gisler-Wüthrich [9] by giving a recursive algorithm for the calculation of the Bayesian estimators. This recursive approach allows for the study of the 
one-year claims development result in the chain ladder method which is of central interest in profit \& loss statements under Solvency II, a discussion is given in Section 3 below and in Merz-Wüthrich [13].

\subsection{Notation and Model Assumptions}

For the notation we closely follow Gisler-Wüthrich [9]. Assume that cumulative claims are denoted by $C_{i, j}>0$, where $i \in\{0, \ldots, I\}$ denotes the accident year and $j \in\{0, \ldots, J\}$ the development year $(I \geq J)$. At time $I$ we have observations in the upper trapezoid

$$
\mathcal{D}_{I}=\left\{C_{i, j}: i+j \leq I\right\},
$$

and we need to predict the future claims in the lower triangle $\left\{C_{i, j}, i+j>I\right.$, $i \leq I\}$. The individual development factors are defined by

$$
Y_{i, j}=\frac{C_{i, j+1}}{C_{i, j}}
$$

for $j \in\{0, \ldots, J-1\}$.

We now define the Bayes chain ladder model considered in Gisler-Wüthrich [9], that is, we assume that the underlying (unknown) chain ladder factors are described by random variables $F_{0}, \ldots, F_{J-1}$, and, given these variables $F_{j}$, we assume that the cumulative claims $C_{i, j}$ satisfy the distribution-free chain ladder model.

\section{Model Assumptions 1.1 (Bayes Chain Ladder Model)}

B1 Conditionally, given $\mathbf{F}=\left(F_{0}, \ldots, F_{J-1}\right)^{\prime}$, the random variables $C_{i, j}$ belonging to different accident years $i \in\{0, \ldots, I\}$ are independent.

B2 Conditionally, given $\mathbf{F}$ and $\left\{C_{i, 0}, C_{i, 1}, \ldots, C_{i, j}\right\}$, the conditional distribution of $Y_{i, j}$ only depends on $F_{j}$ and $C_{i, j}$, and it holds that

$$
\begin{aligned}
E\left[Y_{i, j} \mid \mathbf{F}, C_{i, 0}, C_{i, 1}, \ldots, C_{i, j}\right] & =F_{j}, \\
\operatorname{Var}\left[Y_{i, j} \mid \mathbf{F}, C_{i, 0}, C_{i, 1}, \ldots, C_{i, j}\right] & =\frac{\sigma_{j}^{2}\left(F_{j}\right)}{C_{i, j}} .
\end{aligned}
$$

B3 The random variables $F_{0}, F_{1}, \ldots, F_{J-1}$ are independent.

We give brief model interpretations here, for an extended discussion we refer to Section 3 in Gisler-Wüthrich [9]. 


\section{Remarks.}

- The true (unknown) chain ladder factors are modelled stochastically by the choice of a prior distribution for $F_{j}$. This prior distribution can have different meanings: (i) If the prior distribution is chosen by a pure expert choice then the prior distribution simply reflects the expert's uncertainty about the true underlying chain ladder factors. (ii) If we have claims data sets of similar individual portfolios we collect them into a collective portfolio. Typical situations are: within a company we have the same line of business in different geographic regions (see Example 5.1 below), or different companies run the same line of business and the prior distribution then reflects market information, e.g., specified by the regulator (see Example 5.3 below). For the modelling of different individual portfolios under (ii), one typically assumes that the generic risk parameters are a priori i.i.d. On the other hand business volume may freely vary (Model Assumptions 1.1 are only stated for a single portfolio). (iii) If there is no prior knowledge on the chain ladder factors one chooses uninformative priors for $F_{j}$ (see Section 4.3 below).

- In Gisler-Wüthrich [9] we have seen that the Bayesian chain ladder framework leads to a natural approach for the estimation of the prediction uncertainty. For uninformative priors one obtains an estimate of the conditional mean square error of prediction for the classical chain ladder algorithm. The resulting formula is different but similar to the Mack [12] formula.

- Note that the conditional variance $\sigma_{j}^{2}\left(F_{j}\right)$ in Model Assumptions $1.1 \mathrm{~B} 2$ is a function of $F_{j}$.

Under Model Assumptions 1.1 we can calculate the Bayesian estimator for $F_{j}$, given the observations $\mathcal{D}_{I}$ (using the posterior distribution). This can be done analytically in closed form in the so-called "exponential family and conjugate priors" case (exact credibility case), see Section 6 in Gisler-Wüthrich [9], however in most other cases this can not be done. In such other situations one can either apply numerical methods like Markov chain Monte Carlo methods (see Asmussen-Glynn [2] and Gilks et al. [8]) or one can restrict the class of estimators to credibility estimators (for details we refer to Section 4 in GislerWüthrich [9]). Here, we consider such credibility estimators. We note that the credibility estimators coincide with the Bayesian estimators from the exact credibility case (see Section 6 in Gisler-Wüthrich [9]).

\section{Definition 1.2.}

The credibility based chain ladder predictor for the cumulative claim $C_{i, k}, k>$ $I-i$, at time $I$ is given by (see Definition 4.1 in Gisler-Wüthrich [9])

$$
\widehat{\widehat{C}}_{i, k}^{(I)}=C_{i, I-i} \prod_{j=I-i}^{k-1} \widehat{\widehat{F}}^{(I)},
$$


where the credibility estimate ${\widehat{\widehat{F_{j}}}}^{(I)}$ at time $I$ for $F_{j}$ is given by (see Theorem 4.3 in Gisler-Wüthrich [9])

$$
{\widehat{F_{j}}}^{(I)}=\alpha_{j}^{(I)}{\widehat{F_{j}}}^{(I)}+\left(1-\alpha_{j}^{(I)}\right) f_{j},
$$

where $S_{j}^{[k]}=\sum_{i=0}^{k} C_{i, j}$ and

$$
\begin{aligned}
& \widehat{F}_{j}^{(I)}=\frac{S_{j+1}^{[I-j-1]}}{S_{j}^{[I-j-1]}}=\frac{\sum_{i=0}^{I-j-1} C_{i, j+1}}{\sum_{i=0}^{I-j-1} C_{i, j}}=\sum_{i=0}^{I-j-1} \frac{C_{i, j}}{\sum_{k=0}^{I-j-1} C_{k, j}} Y_{i, j}, \\
& \alpha_{j}^{(I)}=\frac{S_{j}^{[I-j-1]}}{S_{j}^{[I-j-1]}+\sigma_{j}^{2} / \tau_{j}^{2}},
\end{aligned}
$$

and the structural parameters are given by

$$
f_{j}=E\left[F_{j}\right], \sigma_{j}^{2}=E\left[\sigma_{j}^{2}\left(F_{j}\right)\right] \text { and } \tau_{j}^{2}=\operatorname{Var}\left(F_{j}\right) .
$$

Thus, the credibility estimator $\widehat{\widehat{F}}_{j}^{(I)}$ is a weighted average between the classical chain ladder estimator ${\widehat{F_{j}}}^{(I)}$ (based on the information $\mathcal{D}_{I}$ ) and a prior value $f_{j}$. Moreover, it is the optimal estimator among all estimators that are linear in the observations $Y_{i, j}$ (relative to the quadratic loss function). For more on this topic we refer to Bühlmann-Gisler [4]. The conditional mean square error of prediction (MSEP) of the credibility estimator for the chain ladder factors is given in formula (4.10) in Gisler-Wüthrich [9] which reads as

$$
Q_{j}^{(I)}=E\left[\left({\widehat{\widehat{F_{j}}}}^{(I)}-F_{j}\right)^{2} \mid \mathcal{B}_{j}^{(I)}\right]=\alpha_{j}^{(I)} \frac{\sigma_{j}^{2}}{S_{j}^{[I-j-1]}}=\left(1-\alpha_{j}^{(I)}\right) \tau_{j}^{2},
$$

with

$$
\mathcal{B}_{j}^{(I)}=\left\{C_{i, k} \in \mathcal{D}_{I}: k \leq j\right\} .
$$

$\mathcal{B}_{j}^{(I)}$ denotes that first $j+1$ columns of the observed claims development trapezoid $\mathcal{D}_{I}$. These observations serve as a volume measure in the posterior estimation of $F_{j}$. Note also that the random variable $F_{j}$ is independent of $\mathcal{B}_{j}^{(I)}$. This independence is no longer true for $\mathcal{B}_{j+1}^{(I)}$, that is 


$$
d P\left(F_{j} \mid \mathcal{B}_{j}^{(I)}\right)=d P\left(F_{j}\right) \quad \text { and } \quad d P\left(F_{j} \mid \mathcal{B}_{j+1}^{(I)}\right) \neq d P\left(F_{j}\right)
$$

where we write $d P\left(F_{j} \mid \cdot\right)$ for the conditional distributions of $F_{j}$.

\section{Remark 1.3 (Exponential Family and Conjugate Priors, Exact Credibility)}

We define

$$
\tilde{F}_{j}^{(I)}=E\left[F_{j} \mid \mathcal{D}_{I}\right]=E\left[F_{j} \mid \mathcal{B}_{j+1}^{(I)}\right]
$$

$\tilde{F}_{j}^{(I)}$ denotes the Bayesian estimator for $F_{j}$ given the observations $\mathcal{D}_{I}$. Note that in general $\tilde{F}_{j}^{(I)}$ is different from ${\widehat{\widehat{F_{j}}}}^{(I)}$, but in the case of the exponential family and conjugate priors (exact credibility case) they coincide, i.e. $\tilde{F}_{j}^{(I)}=$ ${\widehat{F_{j}}}^{(I)}$, see Theorem 6.4 in Gisler-Wüthrich [9] and Bühlmann-Gisler [4].

\subsection{Prediction Uncertainty}

We measure the prediction uncertainty with the help of the conditional mean square error of prediction. In general assume that at time $I$ we have information $\mathcal{D}_{I}$ and we need to predict the random variable $X$. The conditional mean square error of prediction of a $\mathcal{D}_{I}$-measurable predictor $\widehat{X}^{(I)}$ for $X$ is defined by

$$
\operatorname{msep}_{X \mid \mathcal{D}_{I}}\left(\widehat{X}^{(I)}\right)=E\left[\left(\widehat{X}^{(I)}-X\right)^{2} \mid \mathcal{D}_{I}\right]
$$

Applying this measure of uncertainty to the credibility based chain ladder predictor we obtain (see Theorem 4.4 and Corollary 4.5 in Gisler-Wüthrich [9]).

Result 1.4 (Conditional MSEP, ultimate claim) For $i>I-J$ we have

$$
\begin{aligned}
& \operatorname{msep}_{C_{i, J} \mid \mathcal{D}_{I}}\left({\widehat{\widehat{C}_{i, J}}}^{(I)}\right)=E\left[\left({\widehat{\widehat{C}_{i, J}}}^{(I)}-C_{i, J}\right)^{2} \mid \mathcal{D}_{I}\right] \approx C_{i, I-i} \Gamma_{I-i}^{(I)}+C_{i, I-i}^{2} \Delta_{I-i}^{(I)} \\
& \stackrel{\text { def. }}{=} \widehat{\operatorname{msep}}_{C_{i, J} \mid \mathcal{D}_{I}}\left(\widehat{\widehat{C}}_{i, J}^{(I)}\right),
\end{aligned}
$$

where 


$$
\begin{aligned}
& \Gamma_{I-i}^{(I)}=\sum_{k=I-i}^{J-1}\left\{\prod_{m=I-i}^{k-1}{\widehat{\widehat{F_{m}}}}^{(I)} \sigma_{k}^{2} \prod_{n=k+1}^{J-1}\left(\left(\widehat{\widehat{F}}^{(I)}\right)^{2}+Q_{n}^{(I)}\right)\right\}, \\
& \Delta_{I-i}^{(I)}=\sum_{j=I-i}^{J-1}\left(\left(\widehat{\widehat{F}}^{(I)}\right)^{2}+Q_{j}^{(I)}\right)-\prod_{j=I-i}^{J-1}\left(\widehat{\widehat{F}}^{(I)}\right)^{2} .
\end{aligned}
$$

For aggregated accident years we have

$$
\begin{aligned}
& \operatorname{msep} \sum_{i=I-J+1}^{I} C_{i, J} \mid \mathcal{D}_{I}\left(\sum_{i=I-J+1}^{I} \widehat{{\widehat{C_{i, J}}}^{(I)}}\right)=E\left[\left(\sum_{i=I-J+1}^{I}{\widehat{\widehat{C_{i, J}}}}^{(I)}-\sum_{i=I-J+1}^{I} C_{i, J}\right)^{2} \mid \mathcal{D}_{I}\right] \\
& \approx \sum_{i=I-J+1}^{I} \operatorname{msep}_{C_{i, J} \mid \mathcal{D}_{I}}\left(\widehat{{\widehat{\widehat{C_{i, J}}}}^{(I)}}\right)+2 \sum_{i=I-J+1}^{I} \sum_{k=i+1}^{I} C_{i, I-i} \underset{\widehat{C}_{k, I-i}}{\widehat{(I)}_{I-i}^{(I)}} \\
& \stackrel{\text { def. }}{=} \widehat{\operatorname{msep}} \sum_{i=I-J+1}^{I} C_{i, J} \mid \mathcal{D}_{I}\left(\sum_{i=I-J+1}^{I} \widehat{{\widehat{C_{i, J}}}^{(I)}}\right) \text {. }
\end{aligned}
$$

\section{Remark 1.5 (Exponential Family and Conjugate Priors, Exact Credibility)}

In the exact credibility case the Bayesian estimator coincides with the credibility estimator (see (1.11)) and we have

$$
\begin{aligned}
Q_{j}^{(I)}=E\left[\left(\tilde{F}_{j}^{(I)}-F_{j}\right)^{2} \mid \mathcal{B}_{j}^{(I)}\right] & =E\left[E\left[\left(\tilde{F}_{j}^{(I)}-F_{j}\right)^{2} \mid \mathcal{D}_{I}\right] \mid \mathcal{B}_{j}^{(I)}\right] \\
& =E\left[\operatorname{Var}\left(F_{j} \mid \mathcal{D}_{I}\right) \mid \mathcal{B}_{j}^{(I)}\right] .
\end{aligned}
$$

With (1.8) we therefore obtain

$$
E\left[\operatorname{Var}\left(F_{j} \mid \mathcal{D}_{I}\right) \mid \mathcal{B}_{j}^{(I)}\right]=\alpha_{j}^{(I)} \frac{\sigma_{j}^{2}}{S_{j}^{[I-j-1]}}=\left(1-\alpha_{j}^{(I)}\right) \tau_{j}^{2}
$$

Therefore in many cases $\operatorname{Var}\left(F_{j} \mid \mathcal{D}_{I}\right)$ is approximated/predicted either by $\alpha_{j}^{(I)} \sigma_{j}^{2} /$ $S_{j}^{[I-j-1]}$ or $\left(1-\alpha_{j}^{(I)}\right) \tau_{j}^{2}$. This approximation takes an additional average over $\mathcal{B}_{j}^{(I)}$ and is exact in the normal-normal case. This justifies approximations $\widehat{\operatorname{msep}}_{C_{i, J} \mid \mathcal{D}_{I}}$ and $\widehat{\mathrm{msep}}_{\sum_{i=I-J+1}^{I} C_{i, J} \mid \mathcal{D}_{I}}$ in Result 1.4. In other cases (e.g. in the gammagamma model) one can explicitly calculate $\operatorname{Var}\left(F_{j} \mid \mathcal{D}_{I}\right)$ which then also leads to an exact formula for the conditional MSEP, see Section 9.2.6 in WüthrichMerz [16]. 


\section{Recursive Credibility Formula}

For solvency considerations one needs to study the updating process from time $I$ to $I+1$, i.e. the change in the predictors by the increase of information $\mathcal{D}_{I} \mapsto$ $\mathcal{D}_{I+1}$, that is, when we add a new diagonal to our observations. Therefore, it seems natural to understand the updating and estimation procedure recursively. Early versions of recursive credibility estimation go back to GerberJones [7], Sundt [15] and Kremer [11].

Theorem 2.1 (Recursive Credibility Formula) For $I>j$ we have

${\widehat{F_{j}}}^{(I)}=\beta_{j}^{(I)} Y_{I-j-1, j}+\left(1-\beta_{j}^{(I)}\right) \widehat{\widehat{F}}^{(I-1)}=\widehat{\widehat{F}}^{(I-1)}+\beta_{j}^{(I)}\left(Y_{I-j-1, j}-\widehat{\widehat{F}}^{(I-1)}\right)$,

$Q_{j}^{(I)}=\left(1-\beta_{j}^{(I)}\right) Q_{j}^{(I-1)}$,

where ${\widehat{\widehat{F_{j}}}}^{(I)}=f_{j}, Q_{j}^{(j)}=\tau_{j}^{2}$ and for $I>j$

$$
\beta_{j}^{(I)}=\frac{C_{I-j-1, j}}{C_{I-j-1, j}+\sigma_{j}^{2} / Q_{j}^{(I-1)}} .
$$

Proof. We prove the claim by induction. Assume $I=j+1$, then $\alpha_{j}^{(I)}=\beta_{j}^{(I)}$ and $\widehat{F}_{j}^{(I)}=Y_{0, j}$ which implies that the claim is true for $I=j+1$.

Induction step: Assume that the claim holds true for $I-1 \geq j+1$. We prove that it holds also true for I. From (1.6) and (1.8) we obtain

$$
Q_{j}^{(I)}=\frac{\sigma_{j}^{2}}{S_{j}^{[I-j-1]}+\sigma_{j}^{2} / \tau_{j}^{2}} .
$$

This implies

$$
\frac{Q_{j}^{(I)}}{Q_{j}^{(I-1)}}=1-\frac{C_{I-j-1, j}}{S_{j}^{[I-j-1]}+\sigma_{j}^{2} / \tau_{j}^{2}} .
$$

Thus, there remains to show that the right-hand side is equal to $1-\beta_{j}^{(I)}$ in order to prove the recursive statement for $Q_{j}^{(I)}$. Note that

$$
C_{I-j-1, j}+\sigma_{j}^{2} / Q_{j}^{(I-1)}=S_{j}^{[I-j-1]}+\sigma_{j}^{2} / \tau_{j}^{2},
$$

which implies

$$
\beta_{j}^{(I)}=\frac{C_{I-j-1, j}}{C_{I-j-1, j}+\sigma_{j}^{2} / Q_{j}^{(I-1)}}=\frac{C_{I-j-1, j}}{S_{j}^{[I-j-1]}+\sigma_{j}^{2} / \tau_{j}^{2}} .
$$


Moreover, using the induction assumption for the credibility chain ladder factor

$$
\begin{aligned}
& \beta_{j}^{(I)} Y_{I-j-1, j}+\left(1-\beta_{j}^{(I)}\right){\widehat{\widehat{F_{j}}}}^{(I-1)} \\
& =\frac{C_{I-j-1, j+1}}{S_{j}^{[I-j-1]}+\sigma_{j}^{2} / \tau_{j}^{2}}+\left(1-\beta_{j}^{(I)}\right)\left(\alpha_{j}^{(I-1)}{\widehat{F_{j}}}^{(I-1)}+\left(1-\alpha_{j}^{(I-1)}\right) f_{j}\right) \\
& =\frac{C_{I-j-1, j+1}}{S_{j}^{[I-j-1]}+\sigma_{j}^{2} / \tau_{j}^{2}}+\left(1-\beta_{j}^{(I)}\right)\left(\frac{S_{j+1}^{[I-j-2]}}{S_{j}^{[I-j-2]}+\sigma_{j}^{2} / \tau_{j}^{2}}+\left(1-\alpha_{j}^{(I-1)}\right) f_{j}\right) \\
& =\frac{C_{I-j-1, j+1}}{S_{j}^{[I-j-1]}+\sigma_{j}^{2} / \tau_{j}^{2}}+\frac{S_{j+1}^{[I-j-2]}}{S_{j}^{[I-j-1]}+\sigma_{j}^{2} / \tau_{j}^{2}}+\left(1-\beta_{j}^{(I)}\right)\left(1-\alpha_{j}^{(I-1)}\right) f_{j} \\
& =\alpha_{j}^{(I)}{\widehat{F_{j}}}^{(I)}+\left(1-\beta_{j}^{(I)}\right)\left(1-\alpha_{j}^{(I-1)}\right) f_{j}={\widehat{F_{j}}}^{(I)} .
\end{aligned}
$$

This proves the claim of the theorem.

Corollary 2.2 We have seen that

$$
\beta_{j}^{(I+1)}=\frac{C_{I-j, j}}{S_{j}^{[I-j]}+\sigma_{j}^{2} / \tau_{j}^{2}},
$$

and $\beta_{j}^{(I+1)}$ is $\mathcal{D}_{I}$-measurable.

\section{Remarks 2.3}

- Note that the proof of the theorem is somehow solving the problem by "brute force". It is well-known in credibility theory (see, for example, Sundt [15] or Theorem 9.6 and the successive remark in Bühlmann-Gisler [4]) that we could also give a credibility argument saying that we look for the optimal $\beta_{j}^{(I)}$ that minimizes

$$
\begin{aligned}
& Q_{j}^{(I)}=E\left[\left({\widehat{\widehat{F_{j}}}}^{(I)}-\left.F_{j}\right|^{2} \mid \mathcal{B}_{j}^{(I)}\right]\right. \\
& =E\left[\left(\beta_{j}^{(I)}\right)^{2}\left(Y_{I-j-1, j}-F_{j}\right)^{2} \mid \mathcal{B}_{j}^{(I)}\right]+E\left[\left(1-\beta_{j}^{(I)}\right)^{2}\left({\widehat{\widehat{F_{j}}}}^{(I-1)}-F_{j}\right)^{2} \mid \mathcal{B}_{j}^{(I)}\right] \\
& =\left(\beta_{j}^{(I)}\right)^{2} \frac{\sigma_{j}^{2}}{C_{I-j-1, j}}+\left(1-\beta_{j}^{(I)}\right)^{2} Q_{j}^{(I-1)},
\end{aligned}
$$


where the second equality holds due to the independence of different accident years and unbiasedness. This minimization then leads exactly to the result given in Theorem 2.1 since we consider credibility estimators that are linear in the observations $Y_{i, j}$.

- With Theorem 2.1 we have found a second way to calculate the credibility estimator for the chain ladder factors as well as the ingredients for Result 1.4 which gives the credibility based conditional MSEP estimation for the full development period. The recursive algorithm allows however to get more. It is the key for the derivation of estimates for the one-year claims development result which takes into consideration the updating procedure of information $\mathcal{D}_{I} \mapsto \mathcal{D}_{I+1}$. This is discussed in the next section.

- Note that $\beta_{j}^{(I)}$ given in (2.1) is sometimes not so convenient since one needs first to calculate $Q_{j}^{(I-1)}$. Corollary 2.2 gives a second more straightforward representation.

\section{One-Year Claims Development Result}

In the Solvency II framework the time period under consideration is one year. Henceforth, insurance companies need to study possible shortfalls in their profit \& loss statement and in their balance sheet on a one-year time horizon. For claims reserving, this means that the companies need to study possible changes in their claims reserves predictions when updating the information from $\mathcal{D}_{I} \mapsto \mathcal{D}_{I+1}$. Hence, we assume that we consider "best estimate" predictors for the ultimate claim $C_{i, J}$, both at time $I$ and with updated information at time $I+1$. The credibility based chain ladder predictors are then given by

$$
\begin{aligned}
& \widehat{\widehat{C}}_{i, J}^{(I)}=C_{i, I-i} \prod_{j=I-i}^{J-1} \widehat{\widehat{F}}^{(I)}, \\
& {\widehat{\widehat{C_{i, J}}}}^{(I+1)}=C_{i, I-i+1} \prod_{j=I-i+1}^{J-1}{\widehat{\widehat{F}_{j}}}^{(I+1)}=C_{i, I-i} Y_{i, I-i} \prod_{j=I-i+1}^{J-1} \widehat{\widehat{F}}^{(I+1)} .
\end{aligned}
$$

These two predictors of the ultimate claim $C_{i, J}$ yield the claims reserves estimates $\widehat{R}_{i}^{(I)}$ and $\widehat{R}_{i}^{(I+1)}$ at times $I$ and $I+1$, when we subtract the latest observed cumulative payments at times $I$ and $I+1$, respectively. The claims reserves $\widehat{R}_{i}^{(I)}$ are often called the opening reserves for accounting year $I+1$ and $\widehat{R}_{i}^{(I+1)}$ the closing reserves at the end of this accounting year (see, e.g., Ohlsson-Lauzeningks [14]). The one-year claims development result for accident year $i$ at time $I+1$ analyzes possible changes in this update of predictions of ultimate claims. It is given by (see Merz-Wüthrich [13], formula (2.19))

$$
\widehat{\operatorname{CDR}}_{i}(I+1)={\widehat{\widehat{C}_{i, J}}}^{(I)}-{\widehat{\widehat{C_{i, J}}}}^{(I+1)} \text {. }
$$


This is a random variable viewed from time $I$ and it is known at time $I+1$. In the one-year solvency view we need to study its volatility in order to determine the uncertainty in the annual profit $\&$ loss statement position "loss experience prior accident years" (see Table 1 for an example).

TABLE 1

EXAMPLE OF A PROFIT \& LOSS STATEMENT INCOME

\begin{tabular}{lcc}
\hline \hline & $\begin{array}{c}\text { budget values } \\
\text { (predictions at time I) }\end{array}$ & $\begin{array}{c}\text { P\&L statement } \\
\text { (observations at time I+ 1) }\end{array}$ \\
\hline a) premiums earned & $4^{\prime} 000^{\prime} 000$ & $4^{\prime} 020^{\prime} 000$ \\
b) claims incurred current accident year & $-3^{\prime} 200^{\prime} 000$ & $-3^{\prime} 250^{\prime} 000$ \\
c) loss experience prior accident years & 0 & $-40^{\prime} 000$ \\
d) underwriting and other expenses & $-1^{\prime} 000^{\prime} 000$ & $-990^{\prime} 000$ \\
e) investment income & $600^{\prime} 000$ & $610^{\prime} 000$ \\
\hline Income before taxes & $400^{\prime} 000$ & $350^{\prime} 000$ \\
\hline \hline
\end{tabular}

That is, positition c) in Table 1 is predicted by 0 at time $I$ (see Proposition 3.1 and (3.4), below) and we have an observed claims development result of $-40^{\prime} 000$ at time $I+1$ which reflects the information update at time $I+1$ (for a more extended discussion we refer to Merz-Wüthrich [13] and Ohlsson-Lauzeningks [14]).

This one-year solvency view is in contrast to the classical claims reserving view, where one studies the uncertainties in the claims reserves over the whole runoff period of the liabilities. Therefore, this Solvency II one-year view has motivated several contributions in the actuarial literature. An early paper was written by De Felice-Moriconi [5]. In De Felice-Moriconi [5] the "year-end obligations" of the insurer (i.e. claims paid plus best estimate reserves at time $I+1$ of the ultimate loss) were considered and their predictive distribution was derived using the over-dispersed Poisson (ODP) model. The approach was referred to as "year-end expectation" (YEE) point of view, as opposed to the "liabilityat-maturity" (LM) approach, which corresponds to the traditional long-term view in loss reserving. The YEE approach with the ODP model has also been used by ISVAP [10] in a field study where solvency capital requirements on a large sample of Italian MTPL companies have been derived. De Felice-Moriconi [6] also applied the YEE approach to the distribution-free chain ladder model. The same formulas were derived independently in Wüthrich et al. [17] for the MSEP of the one-year claims development result and a field study by AISAM-ACME [1] analyzed the numerical results of these one-year claims development result formulas. 
Proposition 3.1 (Expected One-Year Claims Development Result) We have for $i>I-J$

$$
\begin{aligned}
E & {\left[\widehat{\operatorname{CDR}}_{i}(I+1) \mid \mathcal{D}_{I}\right] } \\
& =C_{i, I-i}\left(\prod_{j=I-i}^{J-1} \widehat{\widehat{F}}^{(I)}-\tilde{F}_{I-i}^{(I)} \prod_{j=I-i+1}^{J-1}\left\{{\widehat{F_{j}}}^{(I)}+\beta_{j}^{(I+1)}\left(\tilde{F}_{j}^{(I)}-\widehat{\widehat{F}}^{(I)}\right)\right\}\right),
\end{aligned}
$$

where an empty product is equal to 1.

Proposition 3.1 says that the conditionally expected one-year claims development result is, in general, not equal to 0 , i.e. the Bayesian estimator $\tilde{F}_{j}^{(I)}$ may differ from the credibility estimator ${\widehat{F_{j}}}^{(I)}$. Therefore, one may question the terminology "best estimate" reserves (and also the prediction 0 at time $I$ for positition c) in Table 1). However, in most practical situations this is the best one can do, due to the lack of information that would allow to find $\tilde{F}_{j}^{(I)}, j=0, \ldots$, $J-1$.

\section{Remark 3.2 (Exponential Family and Conjugate Priors, Exact Credibility)}

In the exact credibility case $\widehat{\widehat{F}}_{j}^{(I)}=\tilde{F}_{j}^{(I)}$ we obtain that the expected one-year claims development result is equal to zero, that is,

$$
E\left[\widehat{\operatorname{CDR}}_{i}(I+1) \mid \mathcal{D}_{I}\right]=0 \text {. }
$$

This exactly justifies the prediction 0 of the one-year claims development result in the budget statement.

Proof of Proposition 3.1. The proof is essentially similar to the martingale property of successive conditional expectations (tower (iterativity) property of conditional expectations). Using Theorem 2.1 we find

$$
\begin{aligned}
& E\left[\widehat{\operatorname{CDR}}_{i}(I+1) \mid \mathcal{D}_{I}\right] \\
& =C_{i, I-i}\left(\prod_{j=I-i}^{J-1} \widehat{\widehat{F}}^{(I)}-E\left[Y_{i, I-i} \prod_{j=I-i+1}^{J-1} \widehat{\widehat{F}}^{(I+1)} \mid \mathcal{D}_{I}\right]\right) \\
& =C_{i, I-i}\left(\prod_{j=I-i}^{J-1} \widehat{\widehat{F}}^{(I)}-E\left[Y_{i, I-i} \prod_{j=I-i+1}^{J-1}\left\{\widehat{\widehat{F}}^{(I)}+\beta_{j}^{(I+1)}\left(Y_{I-j, j}-\widehat{\widehat{F}}^{(I)}\right)\right\} \mid \mathcal{D}_{I}\right]\right) .
\end{aligned}
$$


Hence, we need to calculate the last term of the equality above. Note that $\beta_{j}^{(I+1)}$ is $\mathcal{D}_{I}$-measurable. We have using the conditional independence of different accident years

$$
\begin{aligned}
& E\left[Y_{i, I-i} \prod_{j=I-i+1}^{J-1}\left\{\widehat{\widehat{F}}^{(I)}+\beta_{j}^{(I+1)}\left(Y_{I-j, j}-\widehat{\widehat{F}}^{(I)}\right]\right\} \mid \mathcal{D}_{I}\right] \\
& =E\left[E\left[Y_{i, I-i} \mid \mathbf{F}, \mathcal{D}_{I}\right] \prod_{j=I-i+1}^{J-1} E\left[{\widehat{F_{j}}}^{(I)}+\beta_{j}^{(I+1)}\left(Y_{I-j, j}-\widehat{\widehat{F}}_{j}^{(I)}\right] \mid \mathbf{F}, \mathcal{D}_{I}\right] \mid \mathcal{D}_{I}\right] \\
& =E\left[F_{I-i} \prod_{j=I-i+1}^{J-1}\left\{\widehat{\widehat{F}}^{(I)}+\beta_{j}^{(I+1)}\left(F_{j}-\widehat{\widehat{F}}^{(I)}\right]\right\} \mid \mathcal{D}_{I}\right] .
\end{aligned}
$$

Next, we use Theorem 3.2 of Gisler-Wüthrich [9] which says that $F_{j}$ have independent posterior distributions, given $\mathcal{D}_{I}$. Hence the above expression is equal to

$$
\begin{aligned}
& =E\left[F_{I-i} \mid \mathcal{D}_{I}\right] \prod_{j=I-i+1}^{J-1}\left\{{\widehat{F_{j}}}^{(I)}+\beta_{j}^{(I+1)}\left(E\left[F_{j} \mid \mathcal{D}_{I}\right]-{\widehat{F_{j}}}^{(I)}\right)\right\} \\
& =\tilde{F}_{I-i}^{(I)} \prod_{j=I-i+1}^{J-1}\left\{\widehat{\widehat{F}}^{(I)}+\beta_{j}^{(I+1)}\left(\tilde{F}_{j}^{(I)}-{\widehat{\widehat{F_{j}}}}^{(I)}\right)\right\} .
\end{aligned}
$$

This completes the proof.

\section{MSEP of the Claims Development Result}

For the estimation of the conditional MSEP of the crediblity based ultimate claim predictor $\widehat{\widehat{C}}_{i, J}^{(I)}$ only the three quantities ${\widehat{\widehat{F_{j}}}}^{(I)}, \sigma_{j}^{2}$ and $Q_{j}^{(I)}$ play a role in $\Gamma_{I-i}^{(I)}$ and $\Delta_{I-i}^{(I)}$ (see (1.13) and (1.14)). If we want to study the volatility in the one-year claims development $I \rightarrow I+1$ instead of the full development we need to replace $Q_{j}^{(I)}$, given in (1.8), by

$$
D_{j}^{(I)}=E\left[\left({\widehat{F_{j}}}^{(I+1)}-{\widehat{\widehat{F_{j}}}}^{(I)}\right)^{2} \mid \mathcal{B}_{j}^{(I)}\right] .
$$

This, we are going to explain. We start the analysis for a single accident year $i>$ $I-J$, and in a second stage we derive the estimators for aggregated accident years. 


\subsection{Single Accident Years}

For the time being we concentrate on a single accident year $i>I-J$. Our goal is to study the conditional MSEP of the one-year claims development result, that is,

$$
\begin{aligned}
& \operatorname{msep}_{\widehat{\operatorname{CDR}}_{i}(I+1) \mid \mathcal{D}_{I}}(0)=E\left[\left(\widehat{\mathrm{CDR}}_{i}(I+1)-0\right)^{2} \mid \mathcal{D}_{I}\right] \\
& =E\left[\left({\widehat{\widehat{C_{i, J}}}}^{(I)}-{\widehat{\widehat{C_{i, J}}}}^{(I+1)}\right)^{2} \mid \mathcal{D}_{I}\right]=\operatorname{msep}{\widehat{\widehat{C}_{i, J}^{(I+1)}}}_{\mid \mathcal{D}_{I}}\left({\widehat{\widehat{C_{i, J}}}}^{(I)}\right) \text {. }
\end{aligned}
$$

Formula (4.2) says that we predict the position one-year claims development result in the budget statement at time $I$ by 0 (see position c) in Table 1) and we want to measure how much the realization of the one-year claims development result $\widehat{\mathrm{CDR}}_{i}(I+1)$ at time $I+1$ fluctuates around this prediction. Formula (4.2) also explains the difference in terminology used in earlier publications by De Felice-Moriconi [6], where the expression "year end expectation" (YEE) is used instead of claims development result (CDR).

Note that in the exact credibility case $\widehat{\widehat{F}}_{j}^{(I)}=\tilde{F}_{j}^{(I)}$ formula (4.2) gives the posterior variance of the one-year claims development result $\widehat{\mathrm{CDR}}_{i}(I+1)$, given $\mathcal{D}_{I}$. Hence, in analogy to Gisler-Wüthrich [9], formula (4.15), we assume that the credibility estimator $\widehat{\widehat{F}}_{j}^{(I)}$ is a good approximation to the Bayesian estimator $\tilde{F}_{j}^{(I)}$, which provides the following estimator for the conditional mean square error of prediction.

Result 4.1 The conditional MSEP of the one-year claims development result for a single accident year $i \geq I-J+1$ is estimated by

$$
\widehat{\operatorname{msep}}_{\widehat{\operatorname{CDR}}_{i}(I+1) \mid \mathcal{D}_{I}}(0)=C_{i, I-i} \Gamma_{I-i}^{D(I)}+C_{i, I-i}^{2} \Delta_{I-i}^{D(I)},
$$

with

$$
\begin{aligned}
& \Gamma_{I-i}^{D(I)}=\sigma_{I-i}^{2}\left(1+\beta_{I-i}^{(I+1)}\right) \prod_{j=I-i+1}^{J-1}\left(\left({\widehat{F_{j}}}^{(I)}\right)^{2}+D_{j}^{(I)}\right), \\
& \Delta_{I-i}^{D(I)}=\prod_{j=I-i}^{J-1}\left(\left(\widehat{\widehat{F}}^{(I)}\right)^{2}+D_{j}^{(I)}\right)-\prod_{j=I-i}^{J-1}\left({\widehat{F_{j}}}^{(I)}\right)^{2},
\end{aligned}
$$

where $\beta_{j}^{(I+1)}$ is given in Corollary 2.2, $Q_{j}^{(I)}$ is given in (1.8) and $D_{j}^{(I)}$ in Lemma 4.3. 


\section{Remarks 4.2}

- Note that the two terms $\Gamma_{I-i}^{D(I)}$ and $\Delta_{I-i}^{D(I)}$ look very similar to the terms $\Gamma_{I-i}^{(I)}$ and $\Delta_{I-i}^{(I)}$ given in (1.13)-(1.14). $\Gamma_{I-i}^{D(I)}$ only contains the first summand of $\Gamma_{I-i}^{(I)}$ corresponding to the one-year development. However, in the $\Gamma$-term we obtain an additional factor which is given by

$$
1+\beta_{I-i}^{(I+1)}=\frac{C_{i, I-i}+S_{I-i}^{[i]}+\sigma_{I-i}^{2} / \tau_{I-i}^{2}}{S_{I-i}^{[i]}+\sigma_{I-i}^{2} / \tau_{I-i}^{2}} .
$$

- Often one uses the terminology $C_{i, I-i} \Gamma_{I-i}^{D(I)}$ as process variance term and $C_{i, I-i}$ $\Delta_{I-i}^{D(I)}$ as parameter estimation error term. This terminology comes from a frequentist's perspective. In a Bayesian setup this is debatable because there are also other natural splits. Moreover, the intuition of process uncertainty and parameter uncertainty gets even more lost in the one-year claims development view. In the one-year view the process variance components also influence parameter estimation error terms (one period later). Observe that in the derivation of Result 4.1 we are shifting terms between variance components. Therefore one should probably drop this frequentist's terminology. For the time-being we keep it because it may help to give interpretations to the different terms.

In the remainder of this subsection we derive the estimator given in Result 4.1. We start with auxiliary results. The fast reader (not interested into the technical details of the derivation of Result 4.1) can directly jump to Result 4.7 for aggregated accident years.

Lemma 4.3. We have for (4.1)

$$
D_{j}^{(I)}=\left(\beta_{j}^{(I+1)}\right)^{2}\left(\frac{\sigma_{j}^{2}}{C_{I-j, j}}+Q_{j}^{(I)}\right)=\beta_{j}^{(I+1)} Q_{j}^{(I)}=Q_{j}^{(I)}-Q_{j}^{(I+1)} .
$$

Proof. By definition of $\beta_{j}^{(I)}$ (see (2.1)) we have

$$
\beta_{j}^{(I+1)}\left(\frac{\sigma_{j}^{2}}{C_{I-j, j}}+Q_{j}^{(I)}\right)=Q_{j}^{(I)} .
$$

Using Theorem 2.1 we have

$$
D_{j}^{(I)}=\left(\beta_{j}^{(I+1)}\right)^{2} E\left[\left(Y_{I-j, j}-{\widehat{\widehat{F_{j}}}}^{(I)}\right)^{2} \mid \mathcal{B}_{j}^{(I)}\right] .
$$


As $Y_{I-j, j}$ and $\widehat{\widehat{F}}_{j}^{(I)}$ belong to distinct accident years, we get

$$
D_{j}^{(I)}=\left(\beta_{j}^{(I+1)}\right)^{2}\left(\frac{\sigma_{j}^{2}}{C_{I-j, j}}+Q_{j}^{(I)}\right)=\beta_{j}^{(I+1)} Q_{j}^{(I)}=Q_{j}^{(I)}-Q_{j}^{(I+1)} .
$$

This completes the proof of the lemma.

Corollary 4.4 We have the following useful identities

$$
\begin{aligned}
& \frac{\sigma_{I-i}^{2}}{C_{i, I-i}}+Q_{I-i}^{(I)}-D_{I-i}^{(I)}=\frac{\sigma_{I-i}^{2}}{C_{i, I-i}}\left(1+\beta_{I-i}^{(I+1)}\right), \\
& \beta_{I-i}^{(I+1)}\left(\frac{\sigma_{I-i}^{2}}{C_{i, I-i}}+Q_{I-i}^{(I)}\right)-D_{I-i}^{(I)}=Q_{I-i}^{(I)}-D_{I-i}^{(I)}=Q_{I-i}^{(I+1)} .
\end{aligned}
$$

Proof. By definitions (1.8) and (2.1) we have

$$
Q_{j}^{(I)}=\alpha_{j}^{(I)} \frac{\sigma_{j}^{2}}{S_{j}^{[I-j-1]}}=\beta_{j}^{(I)} \frac{\sigma_{j}^{2}}{C_{I-j-1, j}} .
$$

Using the result from Lemma 4.3 we obtain

$$
\frac{\sigma_{I-i}^{2}}{C_{i, I-i}}+Q_{I-i}^{(I)}-D_{I-i}^{(I)}=\frac{\sigma_{I-i}^{2}}{C_{i, I-i}}+Q_{I-i}^{(I+1)}=\frac{\sigma_{I-i}^{2}}{C_{i, I-i}}\left(1+\beta_{I-i}^{(I+1)}\right),
$$

and similarly for the last statement. This completes the proof of the corollary.

Lemma 4.5 We have the following approximation

$$
\begin{aligned}
& E\left[\operatorname{Var}\left(Y_{i, I-i} \prod_{j=I-i+1}^{J-1} \widehat{\widehat{F}}_{j}^{(I+1)} \mid \mathcal{D}_{I}, \widehat{\widehat{F}}_{I-i+1}^{(I+1)}, \ldots,{\widehat{F_{J-1}}}^{(I+1)}\right) \mid \mathcal{D}_{I}\right] \\
& \approx\left(\frac{\sigma_{I-i}^{2}}{C_{i, I-i}}+Q_{I-i}^{(I)}\right) \prod_{j=I-i+1}^{J-1}\left(D_{j}^{(I)}+\left({\widehat{\widehat{F_{j}}}}^{(I)}\right)^{2}\right) .
\end{aligned}
$$

Proof. We have the following equality 


$$
\begin{gathered}
E\left[\operatorname{Var}\left(Y_{i, I-i} \prod_{j=I-i+1}^{J-1} \widehat{\widehat{F}}_{j}^{(I+1)} \mid \mathcal{D}_{I}, \widehat{\widehat{F}}_{I-i+1}^{(I+1)}, \ldots, \widehat{\widehat{F}}_{J-1}^{(I+1)}\right) \mid \mathcal{D}_{I}\right] \\
=E\left[\prod_{j=I-i+1}^{J-1}\left(\widehat{\widehat{F}}^{(I+1)}\right)^{2} \operatorname{Var}\left(Y_{i, I-i} \mid \mathcal{D}_{I}, \widehat{\widehat{F}}_{I-i+1}^{(I+1)}, \ldots, \widehat{\widehat{F}}_{J-1}^{(I+1)}\right) \mid \mathcal{D}_{I}\right] .
\end{gathered}
$$

For $j=I-i+1, \ldots, J-1$ we have (see Theorem 2.1)

$$
\widehat{\widehat{F}}^{(I+1)}=\widehat{\widehat{F}}^{(I)}+\beta_{j}^{(I+1)}\left(Y_{I-j, j}-\widehat{\widehat{F}}^{(I)}\right) \text {. }
$$

Since $\widehat{\widehat{F}}_{j}^{(I)}$ and $\beta_{j}^{(I+1)}$ are $\mathcal{D}_{I}$-measurable, the random variable ${\widehat{\widehat{F_{j}}}}^{(I+1)}$ only depends on $Y_{I-j, j}$, given $\mathcal{D}_{I}$. This implies that

$$
\begin{aligned}
\operatorname{Var}\left(Y_{i, I-i} \mid \mathcal{D}_{I},{\widehat{F_{I-i+1}}}^{(I+1)}, \ldots, \widehat{\widehat{F}}_{J-1}^{(I+1)}\right) & =\operatorname{Var}\left(Y_{i, I-i} \mid \mathcal{D}_{I}, Y_{i-1, I-i+1}, \ldots, Y_{I-J+1, J-1}\right) \\
& =\operatorname{Var}\left(Y_{i, I-i} \mid \mathcal{D}_{I}\right),
\end{aligned}
$$

where the last equality follows from the fact that accident years are conditionally independent, given $\mathbf{F}$, and because the posterior of $F_{I-i}$ does not depend on the observations $Y_{i-1, I-i+1}, \ldots, Y_{I-J+1, J-1}$ (different development periods, see also Theorem 3.2 in Gisler-Wüthrich [9]). This immediately implies

$$
\begin{gathered}
E\left[\operatorname{Var}\left(Y_{i, I-i} \prod_{j=I-i+1}^{J-1} \widehat{\widehat{F}}_{j}^{(I+1)} \mid \mathcal{D}_{I},{\widehat{F_{I-i+1}}}^{(I+1)}, \ldots, \widehat{\widehat{F}}_{J-1}^{(I+1)}\right) \mid \mathcal{D}_{I}\right] \\
=\operatorname{Var}\left(Y_{i, I-i} \mid \mathcal{D}_{I}\right) E\left[\prod_{j=I-i+1}^{J-1}\left(\widehat{\widehat{F}}^{(I+1)}\right)^{2} \mid \mathcal{D}_{I}\right]
\end{gathered}
$$

So we need to estimate these two factors. For the first factor we obtain the approximation

$$
\begin{aligned}
\operatorname{Var}\left(Y_{i, I-i} \mid \mathcal{D}_{I}\right) & =E\left[\operatorname{Var}\left(Y_{i, I-i} \mid \mathbf{F}, \mathcal{D}_{I}\right) \mid \mathcal{D}_{I}\right]+\operatorname{Var}\left(E\left[Y_{i, I-i} \mid \mathbf{F}, \mathcal{D}_{I}\right] \mid \mathcal{D}_{I}\right) \\
& =E\left[\frac{\sigma_{I-i}^{2}\left(F_{I-i}\right)}{C_{i, I-i}} \mid \mathcal{D}_{I}\right]+\operatorname{Var}\left(F_{I-i} \mid \mathcal{D}_{I}\right) \\
& \approx \frac{\sigma_{I-i}^{2}}{C_{i, I-i}}+Q_{I-i}^{(I)}
\end{aligned}
$$


where in the last step we have used the approximation similar to the one described after (1.16)-(1.17). The second factor is approximated as follows (note that different accident years are conditionally independent, given $\mathbf{F}$ )

$$
\begin{aligned}
E\left[\prod_{j=I-i+1}^{J-1}\left(\widehat{\widehat{F}}^{(I+1)}\right)^{2} \mid \mathcal{D}_{I}\right] & =E\left[\prod_{j=I-i+1}^{J-1} E\left[\left({\widehat{\widehat{F_{j}}}}^{(I+1)}\right)^{2} \mid \mathbf{F}, \mathcal{D}_{I}\right] \mid \mathcal{D}_{I}\right] \\
& =\prod_{j=I-i+1}^{J-1} E\left[E\left[\left(\widehat{\widehat{F}}^{(I+1)}\right)^{2} \mid \mathbf{F}, \mathcal{D}_{I}\right] \mid \mathcal{D}_{I}\right] \\
& =\prod_{j=I-i+1}^{J-1} E\left[\left(\widehat{\widehat{F}}^{(I+1)}\right)^{2} \mid \mathcal{D}_{I}\right],
\end{aligned}
$$

where the second step follows from the fact that the product runs only over pairwise different development factors $F_{j}$ and the posterior distributions of $F_{j}$ given $\mathcal{D}_{I}$ are independent (see Theorem 3.2 in Gisler-Wüthrich [9]). Similar to the derivations in Gisler-Wüthrich [9] this last term is now approximated by

$$
\prod_{j=I-i+1}^{J-1} E\left[\left({\widehat{F_{j}}}^{(I+1)}\right)^{2} \mid \mathcal{D}_{I}\right] \approx \prod_{j=I-i+1}^{J-1}\left(D_{j}^{(I)}+\left({\widehat{F_{j}}}^{(I)}\right)^{2}\right) .
$$

This completes the proof.

Lemma 4.6 We have the following approximation

$$
\begin{aligned}
& \operatorname{Var}\left(E\left[Y_{i, I-i} \prod_{j=I-i+1}^{J-1} \widehat{\widehat{F}}^{(I+1)} \mid \mathcal{D}_{I}, \widehat{\widehat{F}}_{I-i+1}^{(I+1)}, \ldots, \widehat{\widehat{F}}_{J-1}^{(I+1)}\right] \mid \mathcal{D}_{I}\right) \\
& \quad \approx\left(\widehat{\widehat{F}}_{I-i}^{(I)}\right)^{2}\left\{\prod_{j=I-i+1}^{J-1}\left(D_{j}^{(I)}+\left(\widehat{\widehat{F}}^{(I)}\right)^{2}\right)-\prod_{j=I-i+1}^{J-1}\left(\widehat{\widehat{F}}_{j}^{(I)}\right)^{2}\right\} .
\end{aligned}
$$

Proof. As in Lemma 4.5 above we find

$$
\begin{aligned}
& \operatorname{Var}\left(E\left[Y_{i, I-i} \prod_{j=I-i+1}^{J-1} \widehat{\widehat{F}}^{(I+1)} \mid \mathcal{D}_{I}, \widehat{\widehat{F}}_{I-i+1}^{(I+1)}, \ldots, \widehat{\widehat{F}}_{J-1}^{(I+1)}\right] \mid \mathcal{D}_{I}\right) \\
& =E\left[Y_{i, I-i} \mid \mathcal{D}_{I}\right]^{2} \operatorname{Var}\left(\prod_{j=I-i+1}^{J-1} \widehat{\widehat{F}}^{(I+1)} \mid \mathcal{D}_{I}\right)
\end{aligned}
$$




$$
=E\left[Y_{i, I-i} \mid \mathcal{D}_{I}\right]^{2}\left(E\left[\prod_{j=I-i+1}^{J-1}\left(\widehat{\widehat{F}}^{(I+1)}\right)^{2} \mid \mathcal{D}_{I}\right]-E\left[\prod_{j=I-i+1}^{J-1} \widehat{\widehat{F}}^{(I+1)} \mid \mathcal{D}_{I}\right]^{2}\right)
$$

But then the claim follows using the same arguments and approximations as in the derivations of Lemma 4.5.

Derivation of Result 4.1. Under the exact credibility approximation ${\widehat{\widehat{F_{j}}}}_{(I)}^{(I)} \tilde{F}_{j}^{(I)}$ we approximate

$$
\begin{aligned}
\operatorname{msep}_{\widehat{\operatorname{CDR}}_{i}(I+1) \mid \mathcal{D}_{I}}(0) & \approx \operatorname{Var}\left(\widehat{\operatorname{CDR}}_{i}(I+1) \mid \mathcal{D}_{I}\right)=\operatorname{Var}\left(\widehat{\widehat{C}}_{i, J}^{(I+1)} \mid \mathcal{D}_{I}\right) \\
& =C_{i, I-i}^{2} \operatorname{Var}\left(Y_{i, I-i} \prod_{j=I-i+1}^{J-1} \widehat{\widehat{F}_{j}^{(I+1)}} \mid \mathcal{D}_{I}\right) .
\end{aligned}
$$

There remains to estimate this last term to get an estimation.

$$
\begin{aligned}
& \operatorname{Var}\left(Y_{i, I-i} \prod_{j=I-i+1}^{J-1} \widehat{\widehat{F}}^{(I+1)} \mid \mathcal{D}_{I}\right) \\
&=E\left[\operatorname{Var}\left(Y_{i, I-i} \prod_{j=I-i+1}^{J-1} \widehat{\widehat{F}}^{(I+1)} \mid \mathcal{D}_{I}, \widehat{\widehat{F}}_{I-i+1}^{(I+1)}, \ldots, \widehat{\widehat{F}}_{J-1}^{(I+1)}\right) \mid \mathcal{D}_{I}\right] \\
&+\operatorname{Var}\left(E\left[Y_{i, I-i} \prod_{j=I-i+1}^{J-1} \widehat{\widehat{F}_{j}^{(I+1)}} \mid \mathcal{D}_{I}, \widehat{\widehat{F}}_{I-i+1}^{(I+1)}, \ldots, \widehat{\widehat{F}}_{J-1}^{(I+1)}\right] \mid \mathcal{D}_{I}\right) .
\end{aligned}
$$

Using Lemmas 4.5 and 4.6 imply that we find the following approximation

$$
\begin{aligned}
& \operatorname{Var}\left(Y_{i, I-i} \prod_{j=I-i+1}^{J-1} \widehat{\widehat{F}}^{(I+1)} \mid \mathcal{D}_{I}\right) \\
& \approx\left(\frac{\sigma_{I-i}^{2}}{C_{i, I-i}}+Q_{I-i}^{(I)}\right) \prod_{j=I-i+1}^{J-1}\left(D_{j}^{(I)}+\left(\widehat{\widehat{F}}^{(I)}\right)^{2}\right) \\
& +\left(\widehat{\widehat{F}_{I-i}}\right)^{(I)}\left\{\prod_{j=I-i+1}^{J-1}\left(D_{j}^{(I)}+\left(\widehat{\widehat{F}_{j}^{(I)}}\right)^{2}\right)-\prod_{j=I-i+1}^{J-1}\left(\widehat{\widehat{F}}_{j}^{(I)}\right)^{2}\right\}
\end{aligned}
$$




$$
\begin{gathered}
=\left(\frac{\sigma_{I-i}^{2}}{C_{i, I-i}}+Q_{I-i}^{(I)}-D_{I-i}^{(I)}\right) \prod_{j=I-i+1}^{J-1}\left(D_{j}^{(I)}+\left({\widehat{F_{j}}}^{(I)}\right)^{2}\right) \\
+\prod_{j=I-i}^{J-1}\left(D_{j}^{(I)}+\left({\widehat{F_{j}}}^{(I)}\right)^{2}\right)-\prod_{j=I-i}^{J-1}\left(\widehat{\widehat{F}}^{(I)}\right)^{2} .
\end{gathered}
$$

Finally, we apply Lemma 4.3 and Corollary 4.4 which provide the estimator in Result 4.1.

\subsection{Aggregated Accident Years}

Our goal is to study the conditional MSEP of aggregated accident years given by

$$
\begin{aligned}
& \operatorname{msep} \sum_{i=I-J+1}^{I} \widehat{\operatorname{CDR}_{i}(I+1)} \mid \mathscr{D}_{I}(0)=E\left[\left(\sum_{i=I-J+1}^{I} \widehat{\operatorname{CDR}}_{i}(I+1)-0\right)^{2} \mid \mathcal{D}_{I}\right] \\
& =\operatorname{msep} \widehat{\sum}_{i=I-J+1}^{I}{\widehat{\widehat{C_{i, J}}}}^{(I+1)} \mid \mathcal{D}_{I}\left(\sum_{i=I-J+1}^{I}{\widehat{\widehat{C_{i, J}}}}^{(I)}\right) \\
& \approx \operatorname{Var}\left(\sum_{i=I-J+1}^{I}{\widehat{\widehat{C}_{i, J}}}^{(I+1)} \mid \mathcal{D}_{I}\right) \\
& =\sum_{i=I-J+1}^{I} \operatorname{Var}\left({\widehat{\widehat{C_{i, J}}}}^{(I+1)} \mid \mathcal{D}_{I}\right)+2 \sum_{I-J+1 \leq i<k \leq I} \operatorname{Cov}\left(\widehat{\widehat{C}}_{i, J}^{(I+1)}, \widehat{\widehat{C}}^{(I+1)} \mid \mathcal{D}_{I}\right),
\end{aligned}
$$

where we have used the same approximation as in (4.5). Hence, in addition to the variance terms we need to estimate the covariance terms between different accident years. We choose $i<k$. Similar to the derivations above we find the approximation

$$
\begin{aligned}
& \operatorname{Cov}\left({\widehat{\widehat{C}_{i, J}}}^{(I+1)}, \widehat{\widehat{C}_{k, J}}(I+1) \mid \mathcal{D}_{I}\right) \\
& \approx C_{i, I-i} C_{k, I-k} \prod_{j=I-k}^{I-i-1} \widehat{\widehat{F}}_{j}^{(I)} \operatorname{Cov}\left(Y_{i, I-i} \prod_{j=I-i+1}^{J-1} \widehat{\widehat{F}}^{(I+1)}, \prod_{j=I-i}^{J-1} \widehat{\widehat{F}}^{(I+1)} \mid \mathcal{D}_{I}\right) .
\end{aligned}
$$

Note that the only difference in the derivation now is that $\operatorname{Var}\left(Y_{i, I-i} \mid \mathcal{D}_{I}\right)$ needs to be replaced by (see also (4.4)) 
$\operatorname{Cov}\left(Y_{i, I-i},{\widehat{F_{I-i}}}^{(I+1)} \mid \mathcal{D}_{I}\right)=\beta_{I-i}^{(I+1)} \operatorname{Var}\left(Y_{i, I-i} \mid \mathcal{D}_{I}\right) \approx \beta_{I-i}^{(I+1)} \frac{\sigma_{I-i}^{2}}{C_{i, I-i}}+D_{I-i}^{(I)}$.

Accounting for $D_{I-i}^{(I)}$ in $\Delta_{I-i}^{D(I)}$ we obtain in complete analogy to the single accident year case the following estimator:

Result 4.7 The conditional MSEP of the one-year claims development result of aggregated accident years is estimated by

$$
\begin{aligned}
\widehat{\operatorname{msep}} \sum_{i=I-J+1}^{I} \widehat{\operatorname{CDR}}_{i}(I+1) \mid \mathcal{D}_{I}(0) & =\sum_{i=I-J+1}^{I} \widehat{\operatorname{msep}}_{\widehat{\operatorname{CDR}}_{i}(I+1) \mid \mathcal{D}_{I}}(0) \\
& +2 \sum_{I-J+1 \leq i<k \leq I} C_{i, I-i} \widehat{\widehat{C}_{k, I-i}^{(I)}}\left(\Delta_{I-i}^{D(I)}+\Phi_{I-i}^{D(I)}\right),
\end{aligned}
$$

with

$$
\Phi_{I-i}^{D(I)}=\beta_{I-i}^{(I+1)} \frac{\sigma_{I-i}^{2}}{C_{i, I-i}} \prod_{j=I-i+1}^{J-1}\left(\left({\widehat{\widehat{F_{j}}}}^{(I)}\right)^{2}+D_{j}^{(I)}\right),
$$

where $\beta_{j}^{(I+1)}$ is given in Corollary 2.2, $Q_{j}^{(I)}$ is given in (1.8) and $D_{j}^{(I)}$ in Lemma 4.3.

\section{Remark 4.8}

- We obtain an additional term $\Phi_{I-i}^{D(I)}$ when aggregating accident years. This difference to the conditional MSEP for the ultimate claim (compare with formula (1.15)) comes from the fact that the process variance in the next accounting year has also an effect on the fluctuation of the chain ladder factor estimates one period later. This again indicates that for the one-year claims development result there is no canonical split into process variance and parameter estimation uncertainty as it is done in the frequentist's approach for the total runoff uncertainty (see also Remark 4.2).

\subsection{Claims Development Result in the Asymptotic Credibility Based Chain Ladder Model and the Classical Chain Ladder Model}

In the classical chain ladder model (see Mack [12]) the chain ladder factors $f_{j}$ are supposed to be deterministic parameters and they are estimated by the chain ladder factor estimates $\widehat{F}_{j}^{(I)}$ (frequentist's approach). This gives the classical chain ladder predictor 


$$
{\widehat{C_{i, J}}}^{C L}=C_{i, I-i} \prod_{k=I-i}^{J-1}{\widehat{F_{k}}}^{(I)},
$$

for the ultimate claim $C_{i, J}$ at time $I$. From the credibility based chain ladder predictor ${\widehat{C_{i, J}}}^{(I)}$ we asymptotically obtain the same estimator if we send $\tau_{j}^{2} \rightarrow \infty$ because in that case $\alpha_{j}^{(I)} \rightarrow 1$ and ${\widehat{F_{j}}}^{(I)} \rightarrow{\widehat{F_{j}}}^{(I)}$, see formulas (1.3) and (1.4). The predictor $\widehat{\widehat{C}_{i, J}}(I)$ for finite $\tau_{j}^{2}<\infty$ is called credibility based chain ladder predictor. The asymptotic predictor for $\tau_{j}^{2}=\infty$ is called asymptotic credibility based chain ladder predictor and it gives the same best estimate reserves as the classical chain ladder predictor ${\widehat{C_{i, J}}}^{C L}$.

For the conditional MSEP of the asymptotic credibility based chain ladder predictor we simply use Results 4.1 and 4.7 with

$$
\alpha_{j}^{(I)}=1 \quad \text { and } \quad \beta_{j}^{(I+1)}=\frac{C_{I-j, j}}{S_{j}^{[I-j]}},
$$

hence

$$
Q_{j}^{(I)}=\frac{\sigma_{j}^{2}}{S_{j}^{[I-j-1]}} \quad \text { and } \quad D_{j}^{(I)}=\frac{C_{I-j, j}}{S_{j}^{[I-j]}} \frac{\sigma_{j}^{2}}{S_{j}^{[I-j-1]}}
$$

Summarizing we obtain the following result.

\section{Result 4.9 (CDR for the Asymptotic Credibility Based CL Predictor)}

(i) Single accident years $i \in\{I-J+1, \ldots, I\}$ :

$$
\widehat{\operatorname{msep}}_{\widehat{\operatorname{CDR}}_{i}(I+1) \mid \mathcal{D}_{I}}(0)=C_{i, I-i} \Gamma_{I-i}^{*}+C_{i, I-i}^{2} \Delta_{I-i}^{*},
$$

with

$$
\begin{aligned}
& \Gamma_{I-i}^{*}=\sigma_{I-i}^{2}\left(1+\frac{C_{i, I-i}}{S_{I-i}^{[i]}}\right) \prod_{j=I-i+1}^{J-1}\left(\left(\widehat{F}_{j}^{(I)}\right)^{2}+\frac{C_{I-j, j}}{S_{j}^{[I-j]}} \frac{\sigma_{j}^{2}}{S_{j}^{[I-j-1]}}\right), \\
& \Delta_{I-i}^{*}=\prod_{j=I-i}^{J-1}\left(\left(\widehat{F}_{j}^{(I)}\right)^{2}+\frac{C_{I-j, j}}{S_{j}^{[I-j]}} \frac{\sigma_{j}^{2}}{S_{j}^{[I-j-1]}}\right)-\prod_{j=I-i}^{J-1}\left(\widehat{F}_{j}^{(I)}\right)^{2} .
\end{aligned}
$$

(ii) Aggregated accident years: 


$$
\begin{aligned}
\widehat{\operatorname{msep}} \sum_{i=I-J+1}^{I} \widehat{\operatorname{CDR}}_{i}(I+1) \mid \mathscr{D}_{I}(0) & =\sum_{i=I-J+1}^{I} \widehat{\operatorname{msep}}_{\widehat{\operatorname{CDR}}_{i}(I+1) \mid \mathscr{D}_{I}}(0) \\
& +2 \sum_{I-J+1 \leq i<k \leq I} C_{i, I-i} \widehat{\widehat{C}_{k, I-i}^{(I)}}\left(\Delta_{I-i}^{*}+\Phi_{I-i}^{*}\right),
\end{aligned}
$$

with

$$
\Phi_{I-i}^{*}=\frac{\sigma_{I-i}^{2}}{S_{I-i}^{[i]}} \prod_{j=I-i+1}^{J-1}\left(\left(\widehat{F}_{j}^{(I)}\right)^{2}+\frac{C_{I-j, j}}{S_{j}^{[I-j]}} \frac{\sigma_{j}^{2}}{S_{j}^{[I-j-1]}}\right) .
$$

The conditional MSEP estimators in Result 4.9 are higher than the conditional MSEP estimators for the claims development result in the classical chain ladder model presented in Results 3.2 and 3.3 in Merz-Wüthrich [13]. One obtains equality only if one linearizes Result 4.9.

For the linearization we assume

$$
\frac{\sigma_{I-i}^{2}}{S_{j}^{[I-j]}} \ll\left({\widehat{F_{j}}}^{(I)}\right)^{2},
$$

which allows for a first order approximation for $\Gamma_{j}^{*}, \Delta_{j}^{*}$ and $\Phi_{j}^{*}$ (this is similar to the approximations used in Merz-Wüthrich [13]). Property (4.12) is in many practical example satisfied. $\Gamma_{I-i}^{*}$ and $\Phi_{I-i}^{*}$ are approximated by

$$
\begin{aligned}
& \tilde{\Gamma}_{I-i}^{*}=\sigma_{I-i}^{2}\left(1+\frac{C_{i, I-i}}{S_{I-i}^{[i]}}\right) \prod_{j=I-i+1}^{J-1}\left(\widehat{F}_{j}^{(I)}\right)^{2}, \\
& \tilde{\Phi}_{I-i}^{*}=\frac{\sigma_{I-i}^{2}}{S_{I-i}^{[i]}} \prod_{j=I-i+1}^{J-1}\left(\widehat{F}_{j}^{(I)}\right)^{2} .
\end{aligned}
$$

For the approximation of $\Delta_{j}^{*}$ we use that for $a_{j}$ positive constants with $1 \gg a_{j}$ we have

$$
\prod_{j=1}^{J}\left(1+a_{j}\right)-1 \approx \sum_{j=1}^{J} a_{j}
$$

where the right-hand side is a lower bound for the left-hand side (see also (A.1) in Merz-Wüthrich [13]). Then $\Delta_{I-i}^{*}$ is approximated by 


$$
\tilde{\Delta}_{I-i}^{*}=\prod_{j=I-i}^{J-1}\left({\widehat{F_{j}}}^{(I)}\right)^{2} \sum_{j=I-i}^{J-1} \frac{C_{I-j, j}}{S_{j}^{[I-j]}} \frac{\sigma_{j}^{2} /\left(\widehat{F}_{j}^{(I)}\right)^{2}}{S_{j}^{[I-j-1]}} .
$$

This then gives the following linearized version of the conditional MSEP estimator in the asymptotic credibility based chain ladder method:

\section{Result 4.10 (Asymptotic Cred. Based CL Method, Linear Approximation)}

(i) Single accident years $i \in\{I-J+1, \ldots, I\}$ :

$$
\widetilde{\operatorname{msep}_{\operatorname{CDR}_{i}(I+1)} \mid \mathscr{D}_{I}}(0)=C_{i, I-i} \tilde{\Gamma}_{I-i}^{*}+C_{i, I-i}^{2} \tilde{\Delta}_{I-i}^{*}
$$

(ii) Aggregated accident years:

$$
\begin{aligned}
\widetilde{\mathrm{msep}} \sum_{i=I-J+1}^{I} \widehat{\operatorname{CDR}}_{i}(I+1) \mid \mathscr{D}_{I}(0) & =\sum_{i=I-J+1}^{I} \widetilde{\operatorname{msep}}_{\widehat{\mathrm{CDR}}_{i}(I+1) \mid \mathscr{D}_{I}}(0) \\
& +2 \sum_{I-J+1 \leq i<k \leq I} C_{i, I-i} \widehat{C_{k, I-i}}\left(\tilde{\Delta}_{I-i}^{*}+\tilde{\Phi}_{I-i}^{*}\right) .
\end{aligned}
$$

\section{Remark 4.11}

As already mentioned in Remarks 4.2 and 4.8 one should interpret the sums rather than the single components on the right-hand side of (4.17) and (4.18). Doing so we obtain

$$
\begin{aligned}
& \widetilde{\operatorname{msep}}_{\widehat{C D R}_{i}(I+1) \mid \mathcal{D}_{I}}(0) \\
& =\left({\widehat{C_{i, J}}}^{C L}\right)^{2}\left[\frac{\sigma_{I-i}^{2} /\left({\widehat{F_{I-i}}}^{(I)}\right)^{2}}{C_{i, I-i}}+\frac{\sigma_{I-i}^{2} /\left({\widehat{F_{I-i}}}^{(I)}\right)^{2}}{S_{I-i}^{[i-1]}}+\sum_{j=I-i+1}^{J-1} \frac{C_{I-j, j}}{S_{j}^{[I-j]}} \frac{\sigma_{j}^{2} /\left(\widehat{F}_{j}^{(I)}\right)^{2}}{S_{j}^{[I-j-1]}}\right],
\end{aligned}
$$

and for the right-hand side of (4.18) we obtain

$$
\begin{aligned}
& \underset{\operatorname{msep}}{\sum_{i=I-J+1}^{I}}{\widehat{\mathrm{CDR}_{i}}}_{i}(I+1)\left|\mathscr{D}_{I}(0)=\sum_{i=I-J+1}^{I} \widetilde{\operatorname{msep}_{\mathrm{CDR}_{i}}(I+1) \mid}\right|_{D_{I}}(0) \\
& +2 \sum_{I-J+1 \leq i<k \leq I} \widehat{C_{i, J}} C L \widehat{C_{k, J}} C L\left[\frac{\sigma_{I-i}^{2} /\left({\widehat{F_{I-i}}}^{(I)}\right)^{2}}{S_{I-i}^{[i-1]}}+\sum_{j=I-i+1}^{J-1} \frac{C_{I-j, j}}{S_{j}^{[I-j]}} \frac{\sigma_{j}^{2} /\left(\widehat{F}_{j}^{(I)}\right)^{2}}{S_{j}^{[I-j-1]}}\right] .
\end{aligned}
$$


Formulas (4.19) and (4.20) are now directly comparable to the Mack [12] formulas in the classical chain ladder model (see also Estimators 3.12 and 3.16 in Wüthrich-Merz [16]). Formulas (4.19) and (4.20) show that the linearly approximated conditional MSEP of the one-year claims development risk is lower than the conditional MSEP of the total runoff risk for the ultimate claim calculated by the classical Mack [12] formulas. From the process variance term in the Mack [12] formula one only considers the first term of the sum for the uncertainty in the one-year claims development result. For the parameter estimation error term (4.19) contains the full first term from the Mack [12] formula whereas all the remaining terms are scaled down by $C_{I-j, j} / S_{j}^{[I-j]} \leq 1$.

\subsection{Important Inequalities}

For the reason of completeness we provide various inequalities that apply to our estimators:

(1) In the credibility based chain ladder approach, i.e. $\tau_{j}^{2}<\infty$ (see Results 1.4 and 4.7), we have

$$
\widehat{\operatorname{msep}} \sum_{i=I-J+1}^{I} \widehat{\operatorname{CDR}}_{i}(I+1)\left|\mathscr{D}_{I}(0) \leq \widehat{\operatorname{msep}} \sum_{i=I-J+1}^{I} C_{i, J}\right| \mathscr{D}_{I}\left(\sum_{i=I-J+1}^{I}{\widehat{\widehat{C_{i, J}}}}^{(I)}\right) \text {. }
$$

This observation requires some calculation and says that the uncertainty of the one-year claims development result is bounded from above by the total runoff uncertainty of the ultimate claims predictors. The formal proof for (4.21) is provided in the Appendix.

(2) Of course (4.21) also applies to the asymptotic credibility based chain ladder case, i.e. for $\tau_{j}^{2} \rightarrow \infty$, which says that the estimator for the MSEP of the one-year claims development result from Result 4.9 is bounded by the MSEP of the total runoff provided by Corollary 5.3 in Gisler-Wüthrich [9], i.e. for $\tau_{j}^{2} \rightarrow \infty$ we obtain

$$
\widehat{\operatorname{msep}} \sum_{i=I-J+1}^{I} \widehat{\operatorname{CDR}}_{i}(I+1)\left|\mathscr{D}_{I}(0) \leq \widehat{\operatorname{msep}} \sum_{i=I-J+1}^{I} C_{i, J}\right| \mathcal{D}_{I}\left(\sum_{i=I-J+1}^{I} \widehat{C_{i, J}} \widehat{C l}^{C L}\right),
$$

where the left-hand side now corresponds to the asymptotic credibility based chain ladder claims development result from Result 4.9.

(3) The linear approximation (4.20) for the asymptotic credibility based chain ladder case satisfies

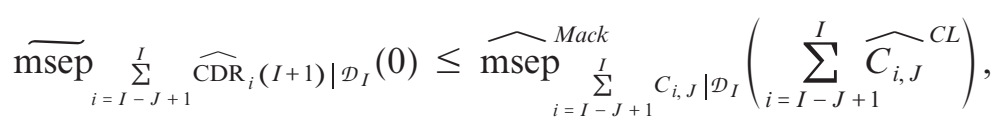


where the right-hand side is the conditional MSEP provided by the classical Mack method [12], the proof is provided in Remark 4.11.

(4) In the asymptotic credibility based chain ladder case, i.e. for $\tau_{j}^{2} \rightarrow \infty$, we find that the linear approximations are lower bounds.

$$
\begin{aligned}
& \widetilde{\operatorname{msep}} \underset{i=I-J+1}{I} \widehat{\operatorname{CDR}}_{i}(I+1)\left|\mathscr{D}_{I}(0) \leq \widehat{\operatorname{msep}} \sum_{i=I-J+1}^{I} \widehat{\operatorname{CDR}}_{i}(I+1)\right| \mathcal{D}_{I}(0), \\
& \widehat{\operatorname{msep}} \underset{i=I-J+1}{M a c k} C_{i, J}^{I} \mid \mathcal{D}_{I}\left(\sum_{i=I-J+1}^{I} \widehat{C_{i, J}}{ }^{C L}\right) \leq \widehat{\operatorname{msep}} \underset{i=I-J+1}{\sum_{i, J}^{I} C_{i, J} \mid \mathcal{D}_{I}}\left(\sum_{i=I-J+1}^{I} \widehat{C_{i, J}} \widehat{C L}^{C L}\right.
\end{aligned}
$$

This follows directly from the derivations.

We illustrate these inequalities in the next section.

\section{EXAMPLES}

We study three examples.

\section{Example 5.1 (Gisler-Wüthrich [9] revisited)}

We revisit the example given in Gisler-Wüthrich [9] with the same parameter choices. The example in Gisler-Wüthrich [9] considers the line of business "building engineering" in different geographic zones in Switzerland, that is, we assume that all these portfolios have a similar behaviour so that prior to any observations we may assume that they satisfy Model Assumptions 1.1 with the same priors.

For the $\sigma_{j}$ we choose the estimators that are taken over the whole portfolio, see Gisler-Wüthrich [9]. The results are illustrated in Table 2.

The case $\alpha_{j}^{(I)}<1$ corresponds to the credibility based chain ladder predictors with an appropriate choice for $\tau_{j}^{2}$. The case $\tau_{j}^{2}=\infty\left(\alpha_{j}^{(I)}=1\right)$ gives the MSEP estimate for the asymptotic credibility based chain ladder predictors, see GislerWüthrich [9] and Subsection 4.3 (Result 4.9, above). Columns (C4), (C6) and (C8) exactly correspond to inequality (4.21) that compares the total runoff risk to the one-year risk in the crediblity based chain ladder case, i.e. for $\tau_{j}^{2}<\infty$. Columns (C3), (C5) and (C7) correspond to inequality (4.22) that does the same consideration in the asymptotic credibility based chain ladder case, i.e. for $\tau_{j}^{2} \rightarrow \infty$. We see that the one-year claims development uncertainty makes almost $80 \%$ of the entire claims development uncertainty.

\section{Example 5.2 (Merz-Wüthrich [13] revisited)}

We revisit the example given in Merz-Wüthrich [13]. There, we have derived estimates for the conditional MSEP of the one-year claims development result in 


\section{TABLE 2}

EXAMPLE [9], REVISITED: CLAIMS RESERVES FROM THE ASYMPTOTIC CREDIBILITY BASED CHAIN LADDER METHOD AND THE CREDIBILITY BASED METHOD, msep ${ }^{1 / 2}$ OVER THE ENTIRE CLAIMS DEVELOPMENT (FULL RUNOFF RISK) AND FOR THE ONE-YEAR CDR.

\begin{tabular}{|c|c|c|c|c|c|c|c|c|}
\hline \multirow[t]{3}{*}{$\mathrm{BU}$} & \multirow{3}{*}{$\begin{array}{r}\text { reserves } \\
\text { CL factors } \\
\widehat{F}_{j}^{(I)}\end{array}$} & \multirow{3}{*}{$\begin{array}{r}\text { reserves } \\
\text { cred. factors } \\
{\widehat{\widehat{F_{j}}}}^{(I)}\end{array}$} & \multicolumn{6}{|c|}{$\mathrm{msep}^{1 / 2}$} \\
\hline & & & \multicolumn{2}{|c|}{ full claims development } & \multicolumn{2}{|c|}{ one-year CDR } & \multicolumn{2}{|c|}{ one-year in $\%$ full } \\
\hline & & & $\begin{array}{l}\text { Cred CL } \\
\alpha_{j}^{(I)}=1 \\
(\mathrm{C} 3)\end{array}$ & $\begin{array}{r}\text { Cred CL } \\
\alpha_{j}^{(I)}<1 \\
(\mathrm{C} 4)\end{array}$ & $\begin{array}{r}\text { Cred CL } \\
\alpha_{j}^{(I)}=1 \\
(\mathrm{C} 5)\end{array}$ & $\begin{array}{r}\text { Cred CL } \\
\alpha_{j}^{(I)}<1 \\
(\mathrm{C} 6)\end{array}$ & $\begin{array}{l}\text { Cred CL } \\
\alpha_{j}^{(I)}=1 \\
(\mathrm{C} 7)\end{array}$ & $\begin{array}{r}\text { Cred CL } \\
\alpha_{j}^{(I)}<1 \\
\quad(\mathrm{C} 8)\end{array}$ \\
\hline A & 486 & 504 & 510 & 498 & 427 & 419 & $83.7 \%$ & $84.1 \%$ \\
\hline B & 235 & 244 & 425 & 402 & 324 & 311 & $76.4 \%$ & $77.4 \%$ \\
\hline $\mathrm{C}$ & 701 & 517 & 566 & 520 & 448 & 415 & $79.2 \%$ & $79.9 \%$ \\
\hline D & $1 ’ 029$ & 899 & 765 & 729 & 598 & 573 & $78.1 \%$ & $78.6 \%$ \\
\hline $\mathrm{E}$ & 495 & 621 & 593 & 584 & 469 & 465 & $79.0 \%$ & $79.5 \%$ \\
\hline $\mathrm{F}$ & 40 & 25 & 163 & 143 & 114 & 100 & $69.7 \%$ & $69.9 \%$ \\
\hline Total & 2'987 & $2^{\prime} 810$ & $1^{\prime} 313$ & 1'254 & $1^{\prime} 038$ & $1^{\prime} 000$ & $79.1 \%$ & $79.7 \%$ \\
\hline
\end{tabular}

\section{TABLE 3}

EXAMPLE [13], REVISITED: CLAIMS RESERVES FROM THE ASYMPTOTIC CREDIBILITY BASED CHAIN LADDER METHOD, THE msep ${ }^{1 / 2}$ OVER THE ENTIRE CLAIMS DEVELOPMENT AND FOR THE ONE-YEAR CDR (RESUlt 4.10 AND Result 4.9).

\begin{tabular}{|c|c|c|c|c|c|}
\hline $\begin{array}{c}\text { acc. year } \\
i\end{array}$ & $\begin{array}{r}\text { reserves } \\
\widehat{C_{i, J}} C L \\
\text { (CL factors) } \\
(\mathrm{C} 1)\end{array}$ & $\begin{array}{r}\text { overall msep }{ }^{1 / 2} \\
\text { formula }(1.15) \\
\text { asym. cred. based CL } \\
\alpha_{j}^{(I)}=1 \\
(\mathrm{C} 2)\end{array}$ & $\begin{array}{r}\text { CDR } \widetilde{\text { msep }} 1 / 2 \\
\text { Result } 4.10 \\
\text { linearized version } \\
\alpha_{j}^{(I)}=1 \\
(\mathrm{C} 3)\end{array}$ & $\begin{array}{r}\text { CDR msep }{ }^{1 / 2} \\
\text { Result } 4.9 \\
\text { non-linearized version } \\
\alpha_{j}^{(I)}=1 \\
(\mathrm{C} 4)\end{array}$ & $\begin{array}{r}\mathrm{CDR} \text { in \% } \\
\text { of (1.15) }\end{array}$ \\
\hline 0 & 0 & & & & \\
\hline 1 & 4'378 & 567 & 567.4 & 567.4 & $100.0 \%$ \\
\hline 2 & 9'348 & 1'566 & $1^{\prime} 488.2$ & $1^{\prime} 488.2$ & $95.0 \%$ \\
\hline 3 & 28'392 & 4'157 & 3'922.6 & 3'922.6 & $94.4 \%$ \\
\hline 4 & $51^{\prime} 444$ & $10^{\prime} 536$ & $9^{\prime} 722.8$ & $9^{\prime} 722.8$ & $92.3 \%$ \\
\hline 5 & $111 ' 811$ & 30’319 & $28^{\prime} 442.5$ & $28^{\prime} 442.5$ & $93.8 \%$ \\
\hline 6 & $187^{\prime} 084$ & $35^{\prime} 967$ & $20 ’ 954.0$ & 20’954.1 & $58.3 \%$ \\
\hline 7 & $411^{\prime} 864$ & $45^{\prime} 090$ & $28 ' 119.3$ & $28 ' 119.3$ & $62.4 \%$ \\
\hline 8 & 1'433'505 & $69^{\prime} 552$ & $53 ' 320.4$ & $53 ’ 320.5$ & $76.7 \%$ \\
\hline Total & 2'237'826 & $108^{\prime} 402$ & $81^{\prime} 080.3$ & $81^{\prime} 080.4$ & $74.8 \%$ \\
\hline
\end{tabular}

the classical CL model which are equal to linearized formulas (4.19)-(4.20), above. We now compare that result with the one obtained from the asymptotic credibility based CL method (non linearized version, see formulas (4.17)-(4.18)). This example also highlights the appropriateness of the linear approximations used in Subsection 4.3. The results are presented in Table 3. 
Columns (C2), (C4) and (C5) correspond to inequality (4.22), that is, in this example the one-year claims development result makes about $75 \%$ of the total runoff risk measured in terms of the conditional MSEP.

Finally, Columns (C3) and (C4) correspond to inequality (4.24) saying that (4.19)-(4.20) (and the method in Merz-Wüthrich [13] for the classical chain ladder model, respectively) give a linear lower bound to Result 4.9. This comes from the fact that in Result 4.9 also higher order terms in the parameter uncertainty are considered. However, the difference in the higher order terms is negligible (as for many real data sets). This is in line with the findings in Buchwalder et al. [3].

\section{Example 5.3 (Italian MTPL Market)}

This example has immediate practical importance in the context of Solvency II where national development factors will be used for companies that are new in the business. Then as they gain data, their own estimates might be credibility weighted with the nation-wide factors. Of course, from a theoretical point of view there is no reason to treat new companies differently from established ones. For branches like Motor Third Party Liability (MTPL) insurance the credibility approach should be used for all companies. As our example will illustrate big companies will automatically have high credibility weights for their own observations.

The example describes a field study on paid losses data of the MTPL market. Complete data of 37 companies was available. That is, these companies have provided $12 \times 12$ sufficiently regular runoff triangles of observations which has allowed for doing our credibility based chain ladder analysis (the data provided was as of end 2006). These $12 \times 12$ triangles were considered to be sufficiently developed in order to do our analysis, moreover we have neglected any possible tail development factor.

For anonymity reasons we have coded the companies according to their business volume. For further protection the business volume of the largest four companies was set equal to their average volume and their ranking is random. The results are given in Table 4. We have used the following abbrevations:

$\% \Delta$ reserves $=\frac{\text { reserves credibility factors }{\widehat{\widehat{F_{j}}}}^{(I)}, \alpha_{j}^{(I)}<1}{\text { reserves CL factors }{\widehat{F_{j}}}^{(I)}, \alpha_{j}^{(I)}=1,}-1$,

$\%$ msep $1=\frac{\text { overall asymptotic credibility } \operatorname{msep}^{1 / 2}, \alpha_{j}^{(I)}=1}{\text { reserves CL factors, }}$,

$\%$ msep $1=\frac{\text { overall credibility } \mathrm{msep}^{1 / 2}, \alpha_{j}^{(I)}<1}{\text { reserves CL factors }}$, 
TABLE 4

EXAMPLE ITALiAn MTPL, THE CAPTION IS GIVEN IN FORMULAS (5.1)-(5.5).

\begin{tabular}{|c|c|c|c|c|c|c|c|c|}
\hline company & $\begin{array}{r}\text { business } \\
\text { volume }\end{array}$ & $\begin{array}{r}\% \Delta \text { reserve } \\
(\%)\end{array}$ & $\begin{array}{r}\text { \%msep } 1 \\
(\%)\end{array}$ & $\begin{array}{r}\text { \%msep } 2 \\
(\%)\end{array}$ & $\begin{array}{r}\% \text { msep CDR } 1 \\
(\%)\end{array}$ & $\begin{array}{r}\text { \%msep CDR } 2 \\
(\%)\end{array}$ & $\begin{array}{r}\frac{\text { \%msep CDR } 1}{\% \text { msep } 1} \\
(\%)\end{array}$ & $\begin{array}{r}\frac{\text { \%msep CDR } 2}{\% \text { msep } 2} \\
(\%)\end{array}$ \\
\hline 1 & 100.0 & 2.5 & 4.03 & 3.99 & 3.24 & 3.21 & 80.4 & 80.3 \\
\hline 2 & 100.0 & -0.3 & 2.90 & 2.86 & 2.36 & 2.33 & 81.4 & 81.4 \\
\hline 3 & 100.0 & -0.6 & 2.41 & 2.38 & 1.98 & 1.96 & 82.3 & 82.3 \\
\hline 4 & 100.0 & -2.5 & 3.45 & 3.37 & 2.85 & 2.78 & 82.6 & 82.5 \\
\hline 5 & 61.8 & -1.1 & 3.66 & 3.58 & 3.04 & 2.97 & 82.9 & 82.8 \\
\hline 6 & 56.9 & 4.2 & 5.54 & 5.50 & 4.50 & 4.47 & 81.2 & 81.2 \\
\hline 7 & 53.0 & 0.9 & 4.52 & 4.44 & 3.70 & 3.63 & 81.8 & 81.8 \\
\hline 8 & 49.4 & 0.6 & 4.60 & 4.52 & 3.82 & 3.75 & 83.1 & 83.0 \\
\hline 9 & 46.2 & 5.1 & 5.61 & 5.52 & 4.59 & 4.52 & 81.8 & 81.8 \\
\hline 10 & 41.6 & 1.4 & 5.32 & 5.22 & 4.36 & 4.28 & 82.0 & 82.0 \\
\hline 11 & 38.6 & -2.3 & 4.47 & 4.29 & 3.65 & 3.50 & 81.8 & 81.6 \\
\hline 12 & 32.4 & -4.0 & 4.68 & 4.47 & 3.88 & 3.70 & 82.9 & 82.8 \\
\hline 13 & 28.5 & 3.8 & 6.80 & 6.58 & 5.47 & 5.30 & 80.5 & 80.5 \\
\hline 14 & 28.3 & -6.6 & 4.75 & 4.32 & 3.81 & 3.47 & 80.3 & 80.3 \\
\hline 15 & 27.7 & 1.3 & 6.40 & 6.23 & 5.17 & 5.04 & 80.7 & 80.9 \\
\hline 16 & 27.3 & 1.1 & 6.97 & 6.70 & 5.80 & 5.56 & 83.3 & 82.9 \\
\hline 17 & 26.6 & 2.1 & 6.10 & 5.86 & 4.99 & 4.79 & 81.8 & 81.7 \\
\hline 18 & 20.8 & 2.4 & 7.03 & 6.72 & 5.69 & 5.45 & 81.0 & 81.1 \\
\hline 19 & 19.3 & 5.3 & 8.71 & 8.40 & 7.07 & 6.81 & 81.1 & 81.1 \\
\hline 20 & 14.0 & -2.6 & 7.90 & 7.35 & 6.49 & 6.03 & 82.1 & 82.1 \\
\hline 21 & 13.1 & -5.5 & 8.05 & 7.42 & 6.54 & 6.04 & 81.2 & 81.3 \\
\hline 22 & 12.3 & -3.2 & 7.97 & 7.23 & 6.50 & 5.91 & 81.6 & 81.8 \\
\hline 23 & 11.8 & 6.8 & 9.87 & 9.31 & 8.07 & 7.63 & 81.7 & 81.9 \\
\hline 24 & 9.2 & -4.5 & 9.73 & 8.39 & 7.95 & 6.87 & 81.7 & 81.9 \\
\hline 25 & 8.3 & -1.4 & 10.21 & 9.15 & 8.43 & 7.58 & 82.6 & 82.8 \\
\hline 26 & 7.8 & -2.2 & 11.21 & 10.22 & 9.25 & 8.45 & 82.5 & 82.7 \\
\hline 27 & 7.4 & -1.7 & 12.83 & 12.02 & 10.86 & 10.10 & 84.6 & 84.0 \\
\hline 28 & 6.8 & -2.6 & 11.87 & 10.49 & 9.59 & 8.55 & 80.8 & 81.4 \\
\hline 29 & 6.1 & -9.7 & 11.00 & 9.27 & 8.90 & 7.58 & 80.9 & 81.7 \\
\hline 30 & 3.5 & 14.7 & 18.02 & 16.54 & 14.78 & 13.75 & 82.0 & 83.1 \\
\hline 31 & 3.4 & 5.2 & 17.23 & 14.99 & 13.92 & 12.35 & 80.8 & 82.4 \\
\hline 32 & 2.6 & -0.7 & 18.73 & 15.68 & 14.89 & 12.85 & 79.5 & 81.9 \\
\hline 33 & 2.5 & 16.3 & 23.11 & 21.46 & 19.10 & 17.97 & 82.6 & 83.7 \\
\hline 34 & 2.2 & -22.1 & 20.83 & 16.64 & 17.53 & 13.98 & 84.2 & 84.0 \\
\hline 35 & 2.0 & -24.0 & 17.01 & 12.96 & 13.87 & 10.72 & 81.5 & 82.8 \\
\hline 36 & 1.8 & 13.8 & 26.16 & 24.03 & 21.54 & 20.16 & 82.4 & 83.9 \\
\hline 37 & 1.8 & 31.9 & 27.79 & 24.14 & 22.25 & 20.15 & 80.1 & 83.5 \\
\hline Total & & -0.2 & 0.96 & 0.93 & 0.78 & 0.76 & 81.8 & 81.8 \\
\hline
\end{tabular}

$\%$ msep CDR $1=\frac{\mathrm{CDR} \text { asymptotic credibility } \mathrm{msep}^{1 / 2}, \alpha_{j}^{(I)}=1}{\text { reserves CL factors, }}$,

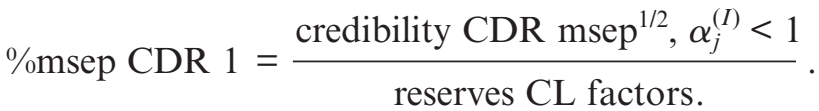




\section{Findings.}

- Especially for smaller companies there is a material difference between the credibility based chain ladder reserves and the chain ladder reserves (column $\% \Delta$ reserves). This comes from the fact that only small credibility weight is attributed to their own observations $\widehat{F}_{j}$ so that their reserves heavily rely on the market parameters $f_{i}$, see (1.4). For the large companies the credibility factors $\alpha_{j}^{(I)}$ were around $94 \%$, whereas for small companies they were in the range of $16 \%$.

- The \%msep's are increasing for decreasing volume. This comes from more diversification and better estimators in larger portfolios. Heuristically, this is a reasonable feature that is also reflected in our observations.

- The \%msep's coming from $\alpha_{j}^{(I)}<1$ are smaller than the ones from $\alpha_{j}^{(I)}=1$. This empirical finding comes from the fact that the prior distribution takes for $\alpha_{j}^{(I)}<1$ some part of the parameter uncertainty.

- The ratios between the uncertainty of the one-year claims development result compared to the total uncertainty of the ultimate claim is around $80 \%$. This corresponds to (4.21)-(4.22). These numerical findings are in line with the field study presented in AISAM-ACME [1].

\section{APPENDIX}

\section{A. Proof of inequality (4.21)}

We start with the derivation for single accident years $i$. Using Corollary 4.4 in the second step we obtain

$$
\begin{aligned}
& \widehat{\operatorname{msep}}_{\widehat{\operatorname{CDR}}_{i}(I+1) \mid \mathcal{D}_{I}}(0)=C_{i, I-i} \Gamma_{I-i}^{D(I)}+C_{i, I-i}^{2} \Delta_{I-i}^{D(I)} \\
& =C_{i, I-i}^{2}\left\{\left[\frac{\sigma_{I-i}^{2}}{C_{i, I-i}}\left(1+\beta_{I-i}^{(I+1)}\right)+\left({\widehat{\widehat{F_{I-i}}}}^{(I)}\right)^{2}+D_{I-i}^{(I)}\right] \prod_{j=I-i+1}^{J-1}\left(\left({\widehat{\widehat{F_{j}}}}^{(I)}\right)^{2}+D_{j}^{(I)}\right)\right. \\
& \left.-\prod_{j=I-i}^{J-1}\left(\widehat{\widehat{F}}^{(I)}\right)^{2}\right\} \\
& =C_{i, I-i}^{2}\left\{\left[\frac{\sigma_{I-i}^{2}}{C_{i, I-i}}+Q_{I-i}^{(I)}+\left({\widehat{\widehat{F_{I-i}}}}^{(I)}\right)^{2}\right] \prod_{j=I-i+1}^{J-1}\left(\left({\widehat{\widehat{F_{j}}}}^{(I)}\right)^{2}+D_{j}^{(I)}\right)\right. \\
& \left.-\prod_{j=I-i}^{J-1}\left({\widehat{\widehat{F_{j}}}}^{(I)}\right)^{2}\right\} \text {. }
\end{aligned}
$$


In the next step we use that

$$
D_{j}^{(I)}=\beta_{j}^{(I+1)} Q_{j}^{(I)} \leq Q_{j}^{(I)}
$$

which implies

$$
\begin{aligned}
& \widehat{\operatorname{msep}}_{\widehat{C D R}_{i}(I+1) \mid \mathcal{D}_{I}}(0)=C_{i, I-i} \Gamma_{I-i}^{D(I)}+C_{i, I-i}^{2} \Delta_{I-i}^{D(I)} \\
& \leq C_{i, I-i}^{2}\left\{\left[\frac{\sigma_{I-i}^{2}}{C_{i, I-i}}+Q_{I-i}^{(I)}+\left(\widehat{\widehat{F}_{I-i}}\right)^{(I)}\right)^{2}\right] \prod_{j=I-i+1}^{J-1}\left(\left(\widehat{\widehat{F}}_{j}^{(I)}\right)^{2}+Q_{j}^{(I)}\right) \\
& \left.-\prod_{j=I-i}^{J-1}\left(\widehat{\widehat{F}}^{(I)}\right)^{2}\right\} \\
& =C_{i, I-i} \sigma_{I-i}^{2} \prod_{j=I-i+1}^{J-1}\left(\left({\widehat{F_{j}}}^{(I)}\right)^{2}+Q_{j}^{(I)}\right)+C_{i, I-i}^{2} \Delta_{I-i}^{(I)} \\
& \leq C_{i, I-i} \Gamma_{I-i}^{(I)}+C_{i, I-i}^{2} \Delta_{I-i}^{(I)}=\widehat{\operatorname{msep}}_{C_{i, J} \mid \mathcal{D}_{I}}\left({\widehat{\widehat{C_{i, J}}}}^{(I)}\right) \text {, }
\end{aligned}
$$

where in the last step we have used that we only need to consider the first term of the sum in $\Gamma_{I-i}^{(I)}$.

Note that we obtain a strict inequality if $\sigma_{j}^{2}>0$ for some $j>I-i$ and all chain ladder factor estimators are strictly positive.

For aggregated accident years we need to consider in addition the covariance terms. For $i<k$ this implies using Corollary 4.4.

$$
\begin{aligned}
& \Delta_{I-i}^{D(I)}+\Phi_{I-i}^{D(I)} \\
& =\left[\beta_{I-i}^{(I+1)} \frac{\sigma_{I-i}^{2}}{C_{i, I-i}}+\left({\widehat{\widehat{F_{I-i}}}}^{(I)}\right)^{2}+D_{I-i}^{(I)}\right] \prod_{j=I-i+1}^{J-1}\left(\left(\widehat{\widehat{F}}_{j}^{(I)}\right)^{2}+D_{j}^{(I)}\right)-\prod_{j=I-i}^{J-1}\left(\widehat{\widehat{F}}_{j}^{(I)}\right)^{2} \\
& =\left[\left(\widehat{\widehat{F}}_{I-i}^{(I)}\right)^{2}+Q_{I-i}^{(I)}\right] \prod_{j=I-i+1}^{J-1}\left(\left(\widehat{\widehat{F}}^{(I)}\right)^{2}+D_{j}^{(I)}\right)-\prod_{j=I-i}^{J-1}\left(\widehat{\widehat{F}}^{(I)}\right)^{2} \\
& \leq \prod_{j=I-i}^{J-1}\left(\left(\widehat{\widehat{F}}_{j}^{(I)}\right)^{2}+Q_{j}^{(I)}\right)-\prod_{j=I-i}^{J-1}\left(\widehat{\widehat{F}}^{(I)}\right)^{2}=\Delta_{I-i}^{(I)} .
\end{aligned}
$$

This completes the proof of inequality (4.21). 


\section{REFERENCES}

[1] AISAM-ACME (2007) AISAM-ACME study on non-life long tail liabilities. Reserve risk and risk margin assessment under Solvency II. October 17, 2007.

[2] Asmussen, S. and Glynn, P.W. (2007) Stochastic Simulation. Springer.

[3] Buchwalder, M., Bühlmann H., Merz, M. and Wüthrich, M.V. (2006) The mean square error of prediction in the chain ladder reserving method (Mack and Murphy revisited). ASTIN Bulletin 36(2), 521-542.

[4] Bühlmann, H. and Gisler, A. (2005) A Course in Credibility Theory and its Applications. Springer, Berlin.

[5] De Felice, M. and Moriconi, F. (2003) Risk based capital in P\&C loss reserving or stressing the triangle. Research Group on "Insurance Companies and Pension Funds", Working Paper n. 1, Rome, December 2003.

[6] De Felice, M. and Moriconi, F. (2006) Process error and estimation error of year-end reserve estimation in the distribution free chain-ladder model. Alef Working Paper, Rome, November 2006.

[7] Gerber, H.U. and Jones, D.A. (1975) Credibility formulas of the updating type. In P.M. Kahn (ed.), Credibility: Theory and Applications. Academic Press.

[8] Gilks, W.R., Richardson, S. and Spiegelhalter, D.J. (1996) Markov Chain Monte Carlo in Practice. Chapman \& Hall, London.

[9] Gisler, A. and WÜthrich, M.V. (2008) Credibility for the chain ladder reserving method. ASTIN Bulletin 38(2), 565-600.

[10] ISVAP (2006) Reserve requirements and capital requirements in non-life insurance. An analysis of the Italian MTPL insurance market by stochastic claims reserving models. Report prepared by De Felice M., Moriconi F., Matarazzo L., Cavastracci S. and Pasqualini S., Rome, October 2006.

[11] Kremer, E. (1982) Credibility for some evolutionary models. Scand. Act. Journal 1982(3-4), 129-142.

[12] MACK, T. (1993) Distribution-free calculation of the standard error of chain ladder reserve estimates. ASTIN Bulletin 23(2), 213-225.

[13] Merz, M. and Wüthrich, M.V. (2008) Modelling the claims development result for solvency purposes. CAS E-Forum, Fall 2008, 542-568.

[14] Ohlsson, E. and LAUZEningks, J. (2008) The one-year non-life insurance risk. Conference Paper, ASTIN Colloquium 2008, Manchester.

[15] Sundt, B. (1981) Recursive credibility estimation. Scand. Act. Journal 1981(1), 3-21.

[16] Wüthrich, M.V. and Merz, M. (2008) Stochastic Claims Reserving Methods in Insurance. Wiley.

[17] Wüthrich, M.V., Merz, M. and Lysenko, N. (2008) Uncertainty in the claims development result in the chain ladder method. Accepted for publication in Scand. Act. J.

HANS BÜHLMANN

Department of Mathematics

ETH Zurich

8092 Zurich, Switzerland

E-Mail:hbuhl@math.ethz.ch

Massimo De Felice

Dipartimento di Studi Sociali, Economici,

Aattuariali e Finanziari

Viale Regina Elena, 295

00161 Roma, Italy

E-Mail:massimo.defelice@uniromal.it 
306 H. BÜHLMANN, M. DE FELICE, A. GISLER, F. MORICONI AND M.V. WÜTHRICH

Alois Gisler

AXA Winterthur Insurance Company

P.O. Box 357

8401 Winterthur, Switzerland

E-Mail: alois.gisler@axa-winterthur.ch

FRANCO MORICONI

Dipartimento di Economia, Finanza e Statistica

Università di Perugia

Via A. Pascoli, 1

06100 Perugia, Italy

E-Mail:moriconi@unipg.it

MARIO V. WÜTHRICH

Department of Mathematics

ETH Zurich

8092 Zurich, Switzerland

E-Mail: wueth@math.ethz.ch 